\title{
LES ATELIERS PRÉCOCES ET LEURS PRODUCTIONS
}

\author{
Armand DESBAT et Martine GENIN
}

\begin{abstract}
Résumé. La dernière partie reprend les principales catégories de céramiques produites régionalement, imitations de sigillée, sigillée, vases à parois fines, gobelets d'Aco, en faisant la synihèse sur leur chronologie, leur typologie ou leur diffusion. Eille aborde également les problèmes plus généraux liés aux ateliers, comme leur genèse, lenur diffusion ou leur déclin. Parmi les résultats on reliendra les points suivants :

- l'étude de la production de Loyasse montre que, contrairement au schéma proposé naguère, il s'agit bién d'un atelier de tradition italique et que sa diffusion est plus importante qu'on ne le supposait;

- à l'inverse le matériel lioré par les fouilles de la Muette ne correspond pas à la grande période de tiffusion des ateliers lyonnais sur le limes;

- Les découvertes récentes montrent des liens étroits entre les ateliers de Vienne et ceux de Lyon, comme l'atteste le transfert de moules de sigillée et de gobelets d'Aco; elles mettent aussi en évidence l'existence d'un atelier de sigillée à Vienne; concernant l'atelier d'Ateivs, la question a pen progressé et sa localisation à Vienne est toujours possible;

- sur la diffusion des ateliers lyonnais, on remarquera que celle-ci ne concerne pas que les sites militaires rhénans, mais également l'ouest et le onord de la ciaule;

- concernant enfin l'arrêt de la production des ateliers de sigillée il faut peut-être chercher sa raison dans la diminution rapide des ressonrces en combustible.
\end{abstract}

Abstract. The study of the production of Loyasse proves that contrany to what was suggested before, it is indeed a workshop of italic tradition and that its distritution is more important that it ruas supposed.

Conversely, the material found in the excavations of la Muette doesn't correspond to the great period of the distribution of the workshops of Lyon on the Limes.

The recent discoveries show narrow links beetween the workshops of Vienne and these of Lyon, which is testified by the transfer of terra sigillata moulds and of Aco-beakers. They also bring to the fore the existence of a workshop of sigillata in Vienne.

As to the Ateivs's workshop, nothing new has been found and it could be localized in Vienne or in Lyon.

About the distribution of the workshops of Lyon, it doesn't only concerm the military sites of the Limes but the West and the North of Gaul as well.

As to the end of the production of terra sigillata, it could be due to the shortage of fuel.

\section{LES CÉRAMIQUES PRÉCOCES À VERNIS NOIR OU ROUGE NON GRÉSÉ}

\section{DÉFINITION}

Les céramiques précoces à revêtement argileux non grésé de couleur noire ou ronge, comme les productions de Loyassc, posent de nombreux problèmes. Le premier est d'abord un problème de définition et de dénomination. Selon les auteurs, des céramiques présentant les mêmes caractéristiques techniques et formelles peuvent être en effet désignées sous le nom de "campaniennes" "imitations de campaniennes " "sigillées noires " "présigillées " ou "imitations de sigillée ». Il est évident que chacune de ces dénominations n'est pas neutre et traduit une interprétation subjective. Celle-ci suppose une filiation, une référence, ou encore une intention prêtée au potier. À dire vrai, ces appellations sont plus ou moins ambiguës, et aucune ne semble parfaitement adéquate pour désigner les céramiques du type de celles de Lovasse. 


\section{"Campanienne"}

Le terme suppose que les productions qui nous intéressent se rattachent culturellement et techniquement aux productions italiques à vernis noir "étrusco-campaniennes ". Or celles-ci se développent à une période où ces dernières sont en déclin. En outre, elles sont de qualité très inférieure aux vraies campaniennes $\mathrm{A}$ et $\mathrm{B}$, et même à la $\mathrm{A}$ tardive, et elles présentent aussi bien des vernis noirs que des vernis rouges ou bruns. En dehors de quelques formes qui reproduisent précisément des types de campanienne, leur répertoire dérive de celui des campaniennes certes, mais au même titre que celui des sigillées archaïques, et comprend les mêmes formes. On voit donc que le terme de "campanienne » n'est pas satisfaisant pour désigner des céramiques hybrides qui se situent à la transition entre les campaniennes et les sigillées du point de vue chronologique, sinon du point de vue technique.

\section{"Imitation de campanienne "}

Cette appellation qui semblerait plus conforme à la qualité technique de ces produits que le terme de "campanienne " a été appliquée dans un premier temps aux productions de Loyasse (Lasfargues, Vertet, 1976, p. 79). Toutefois, les réserves que l'on peut émettre quant au répertoire des formes s'appliquent également ici.

\section{«Sigillée noire "}

Le terme a été créé par M. Schindler (1967) pour désigner certaines céramiques à vernis noir du Magdalensberg, en s'appuyant sur le fait que ces céramiques, se situant à la transition entre les campaniennes et les sigillées, présentaient les mêmes formes que les sigillées. Les analyses ont confirmé qu'une partie de ces sigillées noires provenait d'Arezzo et validé par là même cette appellation (Kaenel, Magetti, 1986). Malgré cela, le terme ne paraît pas approprié à nos céramiques, qui se distinguent trop des sigillées vraies et pour lesquelles une coupure entre les vernis noirs ou bruns et les vernis rouges ne nous semble pas justifiée.

\section{«Pré-sigillée "}

Ce terme créé par N. Lamboglia (1950) était censé désigner une céramique produite pendant la période de transition et correspondant à un passage de la technique des campaniennes à celle des sigillées. Dans l'optique de Lamboglia le "pré " avait donc une connotation à la fois chronologique (antérieur à l'essor des vraies sigillées) et technique (vernis rouge grésé pas encore maîtrisé). Une telle appellation paraîtrait donc mieux convenir aux céramiques précoces type I coyasse que celle de campaniennes ou imitations de campaniennes. Pour cette raison, ce nom a été employé et l'est encore pour désigner les productions de Bram (Passelac, 1986 et 1992). C. M. Wells (Conspectus, 1990; Wells, 1992) a préconisé l'abandon de ce terme dévoyé par un usage intempestif. En effet, certains exemplaires des "pré-sigillées " de Lamboglia se sont avérés faire partie des sigillées orientales, et le terme a même été utilisé pour des céramiques du II" s. avant J.-C.

Toutefois l'argument de Wells ne paraît pas vraiment convaincant; le mauvais usage d'un terme n'est pas un argument pour le supprimer du vocabulaire, si par ailleurs il existe des situations où il est parfaitement approprié. À ce compte, on devrait avoir banni depuis longtemps le terme de sigillée dont le moins que l'on puisse dire, c'est qu'il n'a pas toujours été employé à bon escient. En revanche, là où le nom de "pré-sigillée " ne paraît pas adapté aux productions de type Loyasse, c'est que celles-ci ne correspondent pas à une étape technique vers la fabrication de vernis grésés, mais à des produits réalisés volontairement avec des procédés plus simples. La preuve en est que non seulement des ateliers qui ont produit ces céramiques ne sont pas passés à la fabrication de sigillées, mais encore, que ces productions se poursuivent jusqu'au début du $I^{\text {‘r }}$ s. (en particulier à SaintRomain-en-(ial) parallèlement à celle des vaaies sigillées.

\section{“Imitations de sigillée »}

La dernière dénomination utilisée pour désigner ces productions n'est pas non plus exempte d'ambiguïté. C'est pourtant celle que nous avons retenue pour désigner les productions de Loyasse et de Saint-Romain-enGal, suivant en cela M. Picon et M. Vichy (1974, note 3). L'emploi du terme d'imitation nous paraît toujours se justifier dans la mesure où, dès la période la plus ancienne, ces céramiques s'inspirent d'un répertoire qui est pour l'essentiel celui des premières sigillées, mais avec des procédés de cuisson plus simples, et que le phénomène se prolonge même après le développement des vraies sigillées, avec un répertoire qui continue à copier les formes 
de sigillée avec des procédés techniques plus rudimentaires. Cela ne présuppose pas en revanche qu'il s'agisse de l'ceure de potiers gaulois comme nous le verrons plus loin. C. M. Wells (Conspectus, 1990, p. 25) serait, quant à lui, partisan de conserver le terme d'imitation pour les productions qui seraient le fait de potiers indigènes, "irrespective of quality" comme pour les productions helvètes. Pour les autres, Wells pose la question : "Would it be more appropriate to call their wares "imitations" or to speak of "poor quality sigillata"? " Dans notre esprit, le terme d'imitation insiste au contraire sur l'aspect technique de ces céramiques, en opposition avec les vernis grésés des vraies "arétines" et nous avons choisi de conserver le terme malgré les ambiguïtés qui subsistent.

Aucun des termes que nous venons d'évoquer ne rend vraiment compte à la fois des filiations, des caractéristiques techniques et de la genèse des ateliers, et n'est donc parfaitement adapté pour désigner l'ensemble des productions précoces à vernis noirs ou rouges non grésés. Si l'on voulait avoir unc plus grande cohérence, il faudrait considérer que les ateliers régionaux producteurs de céramiques à vernis non grésé ont commencé en produisant probablement des imitations de campaniennes, puis ont réalisé des "pré-sigillées " et enfin des «imitations de sigillée ". Toutefois, les seuils entre ces différentes catégories sont trop subtils pour que de telles distinctions soient opérantes.

L'existence à I yon et à Vienne d'ateliers ayant produit des imitations de sigillée n'est pas un phénomène isolé. En dehors de l'atelier de Bram (Passelac, 1986), on connaît d'autres ateliers en Narbonnaise, notamment à Narbonne même, mais aussi dans la région, à Villarzens, Villeneuve-la-Comptal, Fleury-d'Aude, Luc-sur-Orbieu (Passelac, 1992), (fig. 86). On suppose également la présence d'ateliers comparables dans la région de Castres, Tarn (Cambon, 1994). Il est probable qu'il y a eu d'autres ateliers de ce type en particulier dans la vallée du Rhône, dont les productions peuvent avoir les mêmes caractéristiques techniques et ne pas se distinguer à l'œil nu. P. Arcelin avait déjà évoqué l'existence, dans la basse vallée du Rhône, de productions inspirées des campaniennes (Arcelin, 1978, p. 120-123). L'étude du mobilier de Glanum a confirmé la présence de céramiques régionales à pâtes claires qui s'inspirent surtout de la forme Lamb. 8 (Arcelin, 1991, p. 218-221). À côté d’exemplaires qui copient les formes campaniennes, existent des vases qui s'apparentent davantage aux productions lyonnaises. C'est le cas pour des bols à parois obliques que leurs caractéristiques trpologiques rapprochent davantage des sigillées que des campaniennes (fig. 87). Les fouilles de Vaison-la-Romaine ont également livré des bols à parois obliques, probablement d'origine locale. Les analyses effectuées sur une dizaine d'exemplaires au Laboratoire

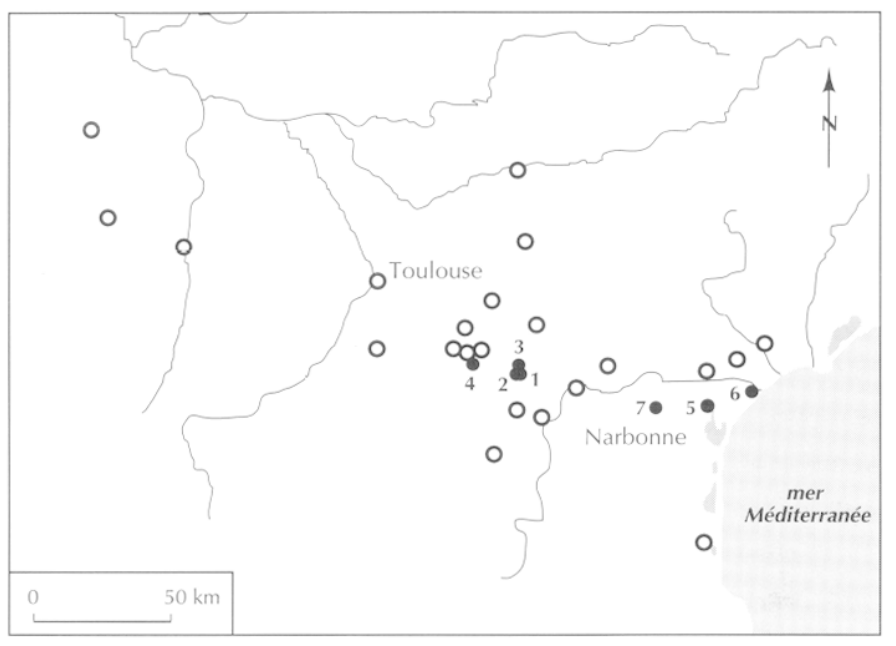

Fig. 86. Carte des ateliers de "pré-sigillée " de Narbonnaise occidentale (d'apress Passelac, 1992) : 1, 2, Bram, vicus; 3, Bram, Villarzens; 4, Villeneuve-la-Comptal, Le Cammazou; 5, Narbonne; 6, Fleury-d'Aude, L'Oustalet; 7, Luc-sur-Orbieu, Le Jouncas;

-, ateliers; ${ }^{\circ}$, principaux sites de diffusion.
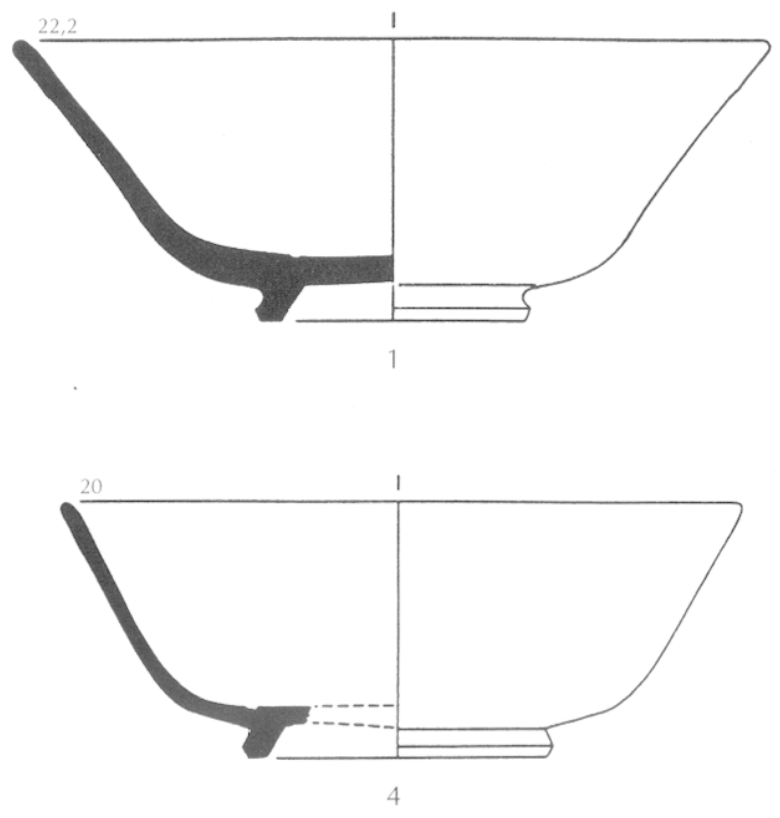

Fig. 87. Exemples de bols à parois obliques en "imitations de campaniennes " (d'après Arcelin, 1991) (échelle: 1/3). 
de céramologie de Lyon ont confirmé l'homogénéité de ces productions et leur différence de composition avec les céramiques lyonnaises et viennoises du même type.

Les parentés techniques entre ces productions, toutes à pâte calcaire, fine, avec des revêtements dont les coloris peuvent varier, constituent l'obstacle majeur pour distinguer, en l'absence d'analyses, les céramiques provenant d'ateliers différents. Il est notamment très difficile de séparer à l'œil nu les productions de Loyasse d'autres productions régionales, voire lyonnaises. Les analyses effectuées sur du matériel provenant de sites de consommation lyonnais, comme celui de la rue des Farges ou du clos du Verbe-Incarné, et que l'on pouvait supposer, au vu de leurs caractéristiques techniques, provenir de l'atelier de Loyasse, ont révélé des compositions différentes de celles de l'atelier, mais proches des compositions de la Muette. Ce résultat inattendu tendrait à prouver, sinon l'existence d'une phase ancienne de production à la Muette, jusqu'ici inconnue, tout au moins l'existence d'autres ateliers du type de celui de Loyasse à Lyon même, probablement situés dans le val de Saône.

De même, les analyses effectuées en Suisse sur des céramiques à vernis noir, désignées comme " campaniennes " mais que l'on peut assimiler à nos "imitations ", ont révélé des compositions proches de celles de la Muette (Kaenel, Magetti, 1986). Ces exemplaires ont été pour cette raison attribués à Lyon et plus précisément à la Muette, alors qu'ils correspondent à une période antérieure à celle de l'activité de l'atelier.

Le matériel livré par les sites lyonnais ayant connu une occupation préaugustéenne, comme le clos du VerbeIncarné, le fossé de Sainte-Croix (Mandy et alii, 1990), le cosidetto sanctuaire de Cybèle ${ }^{16}$, a montré l'existence, dès les années 40-30 avant J.-C., de produits en tous points comparables à ceux de Loyasse sans que l'on puisse déterminer s'il s'agit effectivement de céramiques fabriquées à Lyon. Il est donc tout à fait possible et même probable qu'il ait existé dès la fondation de Lyon des ateliers ayant produit des céramiques de tradition italique, avant l'essor des importations de sigillée.

A. D.

\footnotetext{
16. Les sondages entrepris depuis 1991 à l'emplacement du bâtiment désigné sous le nom de "sancuaire de Cubèle " ont révélé que l'édifice avait été construit au tout début de notre ère et qu'il avait été précédé par deux phases de construction. La première vers 40 avant J.-C., la seconde vers 20 avant J.-C. (Desbat, à paraître).
}

\section{LE RÉPERTOIRE DES FORMES}

Le répertoire des formes pour la période précoce est constitué pour l'essentiel de vases que l'on peut faire dériver des céramiques campaniennes, mais qui appartiennent également au répertoire des premières céramiques à vernis rouge :

- bols hémisphériques,

- plats, assiettes et bols à bord oblique,

- plats, assiettes et bols apparentés aux formes archaïques et précoces de sigillée, en particulier des plats ou des bols à lèvre pendante.

L'atelier de Loyasse a effectivement fabriqué des vases de ces trois groupes mais la production, matérialisée par une seule fosse, de surcroît isolée, ne possède pas a priori une valeur représentative véritablement fiable, comme nous l'avons déjà souligné. Cet atelier rend compte d'une partie seulement de ce qui existait, ce que confirme le mobilier mis au jour à Lyon et dont un échantillon a fait l'objet d'analyses physico-chimiques de pâtes (fig. 88 et 89). Par ailleurs, le répertoire des imitations lyonnaises ressemble sans conteste aux répertoires des autres ateliers, qu'ils soient proches (Saint-Romain-en-Gal) ou éloignés (Bram), mais on ne peut pas parler, pour ces imitations, de répertoire "normalisé " à l'image de celui des sigillées augustéennes d'époque classique (fig. 90). Cela ressort très clairement lorsqu'on observe, par atelier ou par site, le groupe des formes apparentées au service I : ce groupe comprend un très grand nombre de variantes ne formant pas de " types " précisément définissables mais davantage des «familles » de formes; ainsi en est-il en particulier pour les profils de bols se situant à la frontière des formes dites " précoces » et du service IB proprement dit, mais la remarque vaut également pour les vases imitant le service IA.

M. G.

\section{CHRONOLOGIE DES « IMITATIONS " À VERNIS NOIRS OU ROUGES}

Les données fournies par les sites de Lyon et de Vienne, ainsi que les sites de consommation extérieurs permettent de préciser la chronologie de ce type de production. On notera en premier lieu leur absence dans le matériel des fossés du Verbe-Incarné à Lyon (Mandy et alii, 1988 et 1989). Le plus ancien site lyonnais où apparaissent ces productions est le site de Sainte-Croix (Mandy 

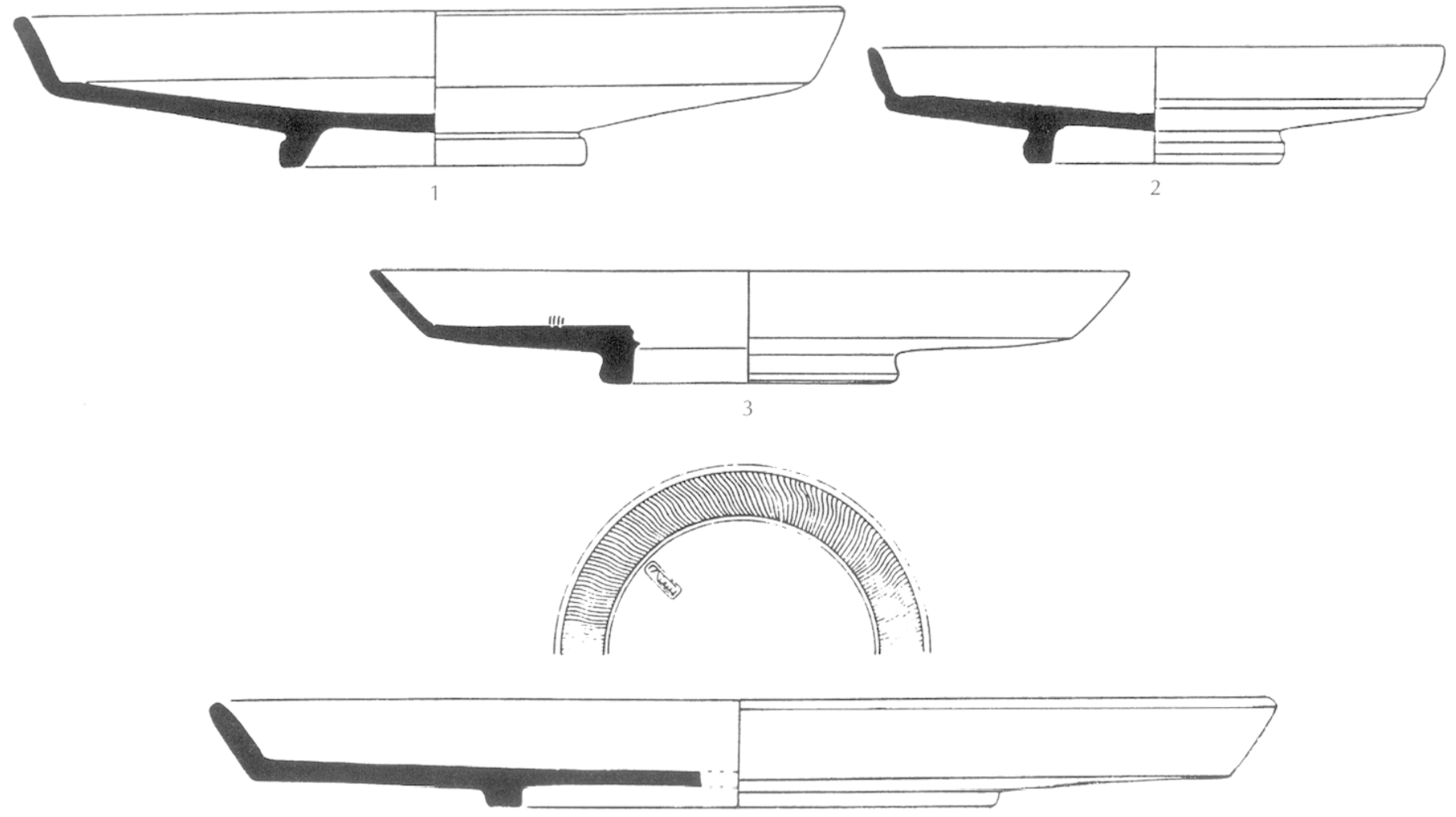

4

[DI
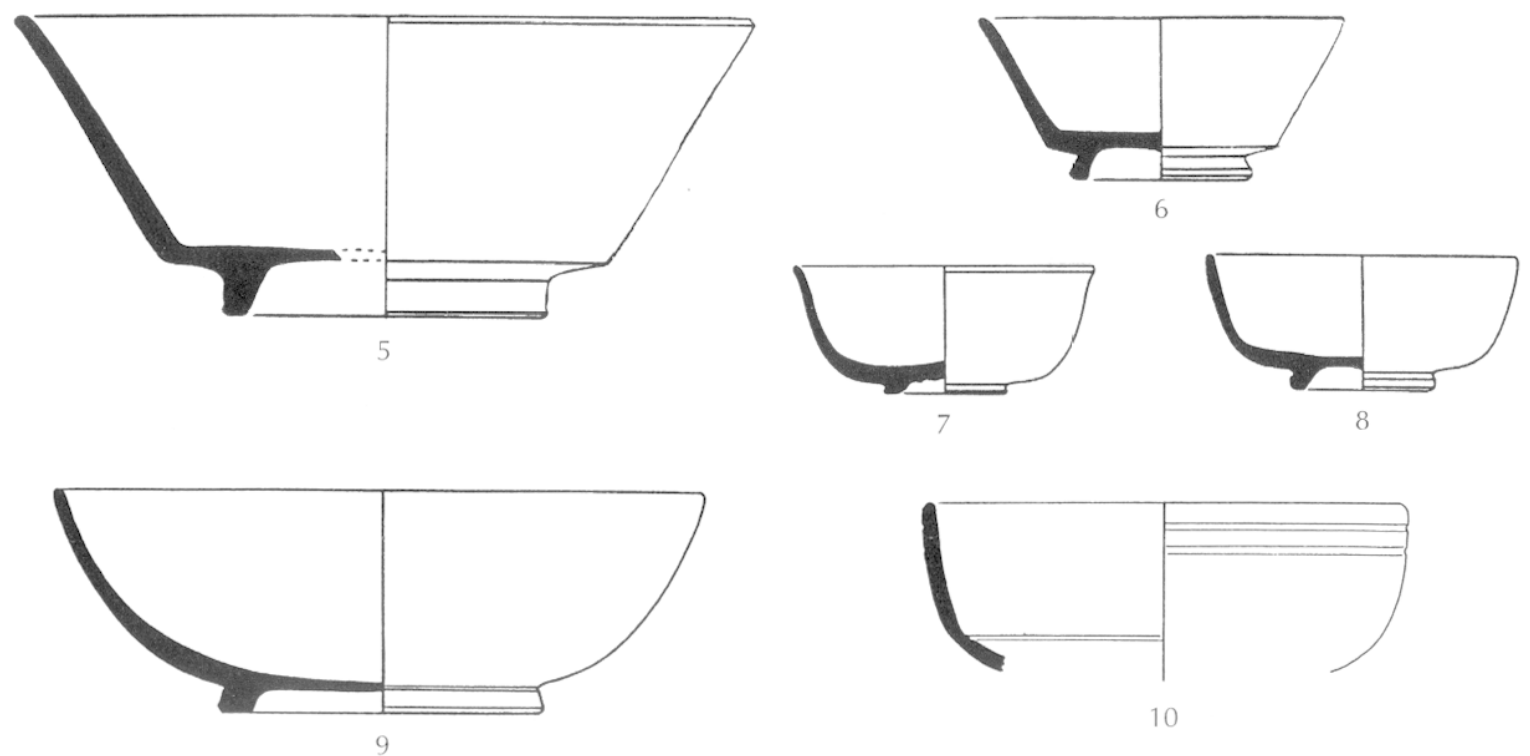

10

Fig. 88. Imitations de sigillée des sites du Verbe-Incarné (1, 2, 4-8) et de la mee des Farges (3, 9, 10) (échelle: 1/3). 

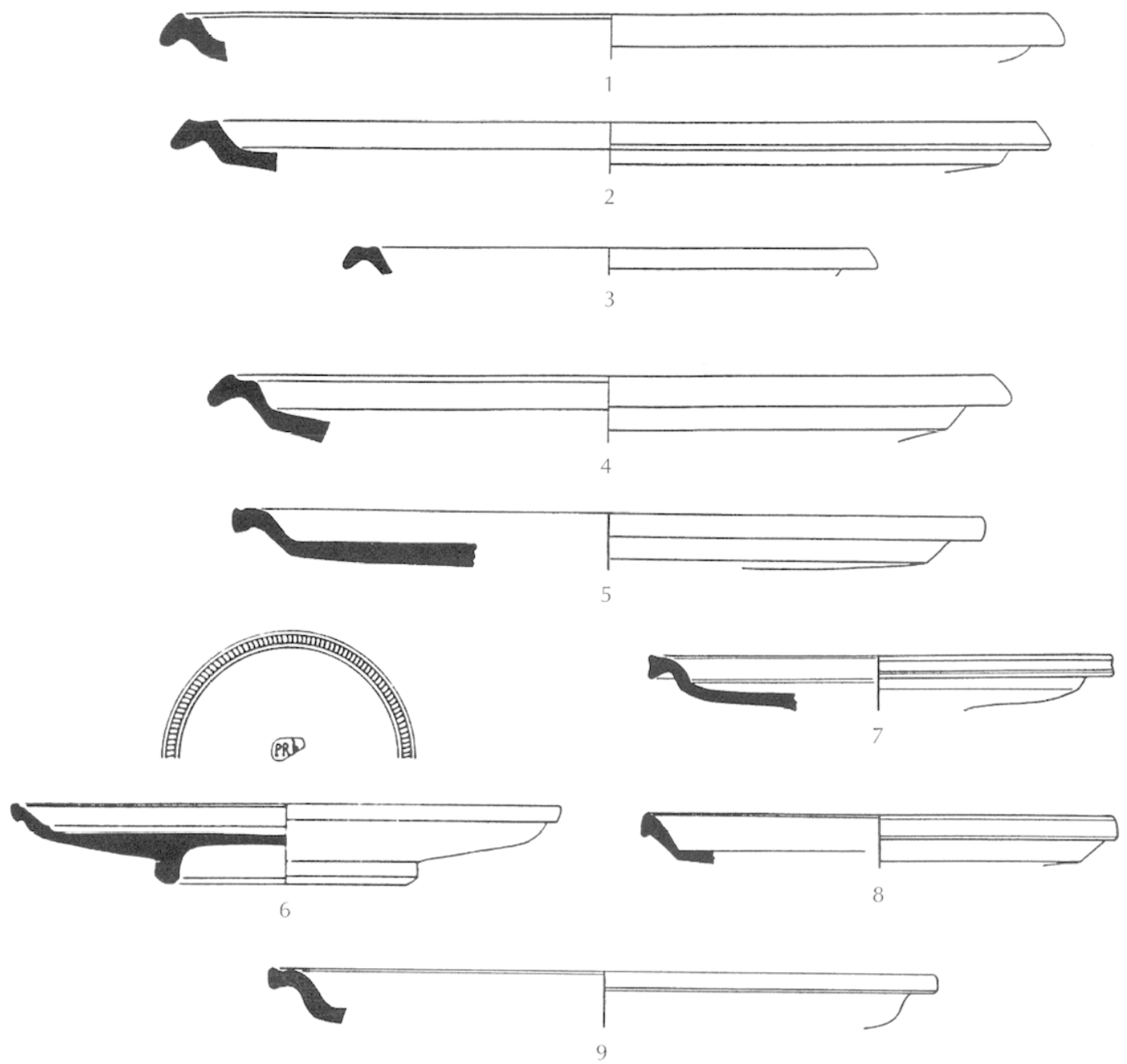

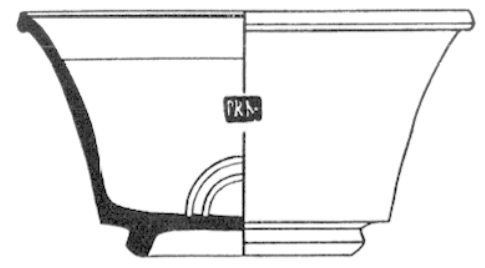

10

U. Ave

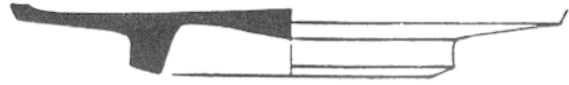

12

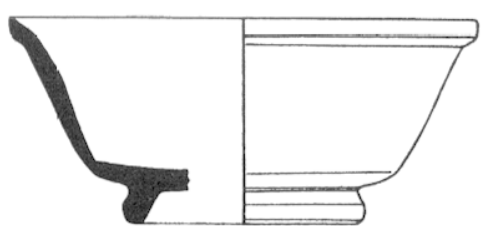

11

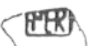

13

Fig. 89. Imitations de sigillée des sites du Verbe-Incarné $(1-4,6,9,10,12,13)$ et de la rue des Farges (5, 7, 8, 11) (échelle: 1/3). 

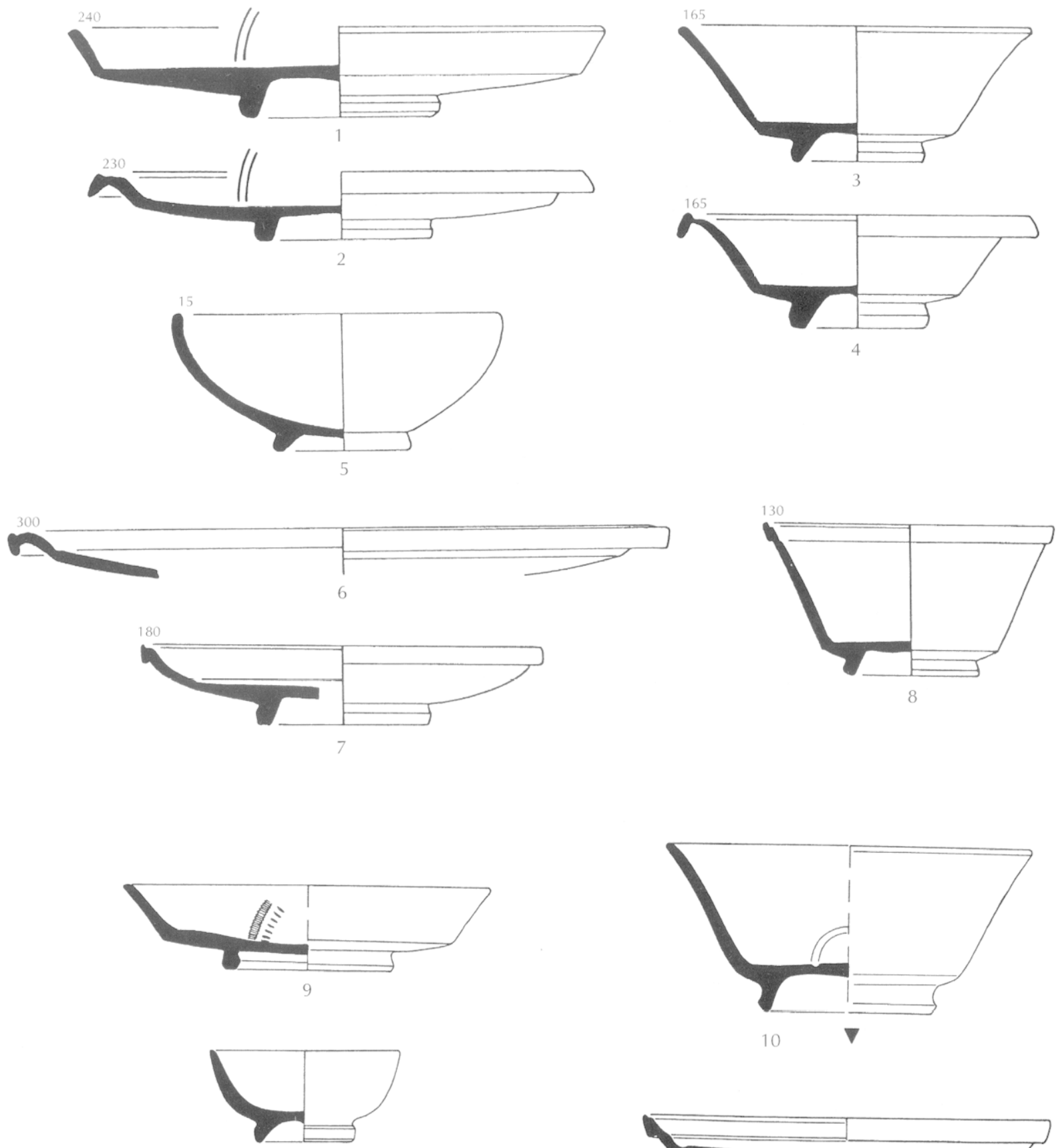

11
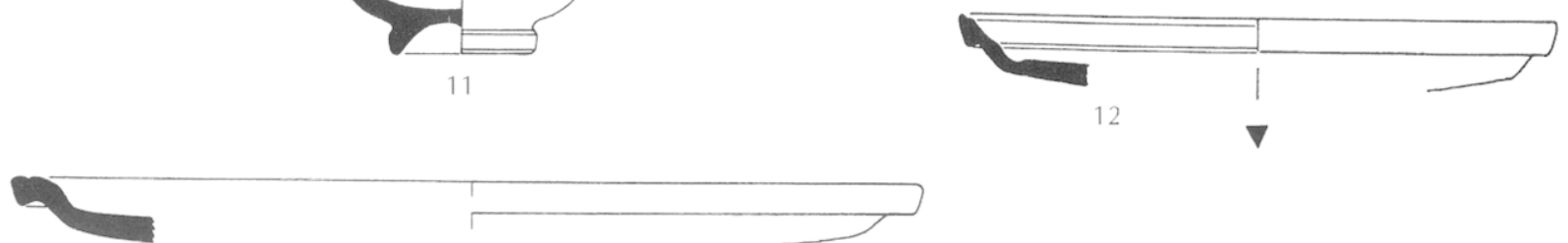

13

Fig. 90. Pré-sigillée de Bram (1-8, d'après Passelac, 1992) et "imitations" de sigillée de Saint-Romain-en-Gal (9-13, d'après Desbat, SazuayGuerraz, 1986) (échelle: 1/3). 
et alii, 1990). Elles y sont associées à de nombreuses amphores Dressel 1, quelques tessons de campanienne, des plats à vernis rouge pompéien. On note l'absence de sigillée. Les formes d'imitations sont représentées par quatre vases dont un plat à lèvre oblique et un bol à paroi oblique. La datation se situerait dans la période 60-40 avant J.-C. plus probablement à l'époque de la fondation. Le second site est celui de la ZAC Saint-Vincent, où des imitations à vernis noir figurent en association avec des campaniennes, des amphores Dressel 1 mais aussi des sigillées précoces, dans la première phase, datées autour de 40 avant J.-C. (Lascoux et alii, 1986, 1987, 1989).

Ces productions apparaissent encore dans les premiers nivcaux lićs à l'urbanisation du site du VerbeIncarné (Desbat et alii, 1989) ainsi que sur le site du "sanctuaire de Cybèle » dans les contextes datés 40-20 avant J.-C. (Desbat, à paraître) et sur celui de Loyasse L3 (Genin, 1994). Sur ces trois sites, elles sont associées à des sigillées arétines précoces et des gobelets d'Aco. En dehors de Lyon, le site de Saint-Romain-en-Gal a fourni également des indications chronologiques précises. Dès le premier horizon (SRG 1), daté 30-20 avant J.-C., elles sont présentes, en association avec des sigillées arétines et des gobelets d'Aco (Desbat, Picon, 1992; Desbat et alii, 1994).

Dans le sud, P. Arcelin en s'appuyant sur de nombreuses références régionales situe la phase de production des imitations dans la seconde moitié du $\mathrm{I}^{\mathrm{wr}} \mathrm{s}$. avant J.-C. : « ce sont uniquement les contextes postérieurs au milieu du $I^{\mathrm{cr}} \mathrm{s}$., plus précisément entre 40 avant et 10 après J.-C., qui contiennent les productions arlésiennes (sic) " (Arcelin, 1991, p. 221). De même, à propos des "céramiques régionales à vernis rouge » trouvées à Nîmes, P.-Y. Genty (1981, p. 106) date la production de la seconde moitié du $\mathrm{I}^{\mathrm{cr}}$ s. avant J.-C.

Au nord de Lyon, on rencontre ces productions notamment à Besançon. Des exemples de productions régionales à vernis noir apparaissent dans la phase 1 (6040 avant J.-C.) (Guilhot et alii, 1992, fig. 140, contexte 4971), la phase 3 (30-1 avant J.-C.) et la phase 4 (1 à 15 après J.-C.). On les rencontre également à Bâle, à la Gas Fabrik, ainsi qu'au Munsterhügel. À la Gas Fabrik, elles apparaissent antérieurement aux premières sigillées, en revanche au Munsterhügel (Fürger-Gunti, 1979), elles sont associées aux sigillées et aux gobelets d'Aco (couche 3 supérieure : $n^{\circ} 239$, pl. 20, à vernis noir, $n^{\circ} 240$, à vernis rouge "pré-sigillèe "; couche 3 inférieure, n“ 358 , pl. 21, pré-sigillée). Des " imitations" à vernis noir ou rouge existent dans d'autres contextes précoces comme les tombes de Goeblingen-Nospelt (Metzler, 1984) ou au Petrisberg (Gocthert-Polaschcck, 1984, pl. 41, 2). Ces deux ensembles pouvant être datés autour des années 3020 avant J.-C.

Tous ces éléments conduisent à placer l'apparition de ces « imitations " vers les années 50 avant J.-C., ou entre 50 et 40 avant J.-C., avant l'essor des importations de vraies sigillées. On a vu cependant que ces productions ne disparaissent pas avec l'essor des sigillées, et se prolongent plus longtemps que la période de fonctionnement de l'atelier de Loyasse. Elles sont toujours présentes dans les contextes classiques où leur pourcentage reste relativement élevé, et souvent en augmentation par rapport aux contextes de la période 40-20 avant J.-C. Ce phénomène est particulièrement net à Saint-Romain-en-Gal (Desbat $e t$ alii, 1994) où l'on peut penser qu'il est dû à la présence d'ateliers locaux dont la production semble s'être prolongée jusqu'au I' s. après J.-C. (Desbat, Savay-Guerraz, 1986). Ce phénomène s'observe également à Besançon (Guilhot et alii, 1992) où les "imitations" représentent un pourcentage plus élevé dans la phase 4 (1-15 après J.-C.) que dans les trois phases précédentes.

Enfin. un des traits majeurs de cette production est son caractère archaïsant : tout se passe comme si le répertoire se trouvait décalé par rapport à celui des sigillées. Ainsi, les formes à parois obliques continuent-elles à être diffusées bien plus tard que les formes correspondantes en sigillée. Ce phénomène a été mis en évidence à SaintRomain-en-Gal (Desbat, 1990b, p. 250 et fig. 4), à Lyon sur les sites de Loyasse L3 (Genin, 1994) et du VerbeIncarné (Genin, 1993). Il se vérifie également sur d'autres sites : à Dangstetten, par exemple, les « imitations " qui comptent 91 vases, contre 386 en sigillée, offrent un faciès légèrement plus ancien que ces dernières; elles ne comprennent pratiquement pas de service IC, mais de nombreux exemples de bols à petites lères, variantes précoces du service IB. De même, les contextes de Saint-Romain-en-Gal montrent le développement des plats et des bols du service II en imitations, plus tard qu'en sigillée, et ceux-ci sont abondants dans les contextes où apparaissent les premières importations de Gaule du Sud.

La production et la commercialisation tardives des formes archaïques sont particulièrement bien illustrées par le dépôt de céramiques de Vienne de l'époque de Claude (Godard, 1992a et b). Non seulement les imitations y sont plus nombreuses que les sigillées, mais 
encore, les formes archaïques représentent-elles $67 \%$ contre $21 \%$ pour le service II et $1,7 \%$ pour les formes récentes (Drag. 24/25 et Drag. 27). Ce phénomène n'est pas propre aux ateliers rhodaniens. Le lot de " pré-sigillée " de Castres (Cambon, 1994) montre le même décalage et cet ensemble daté de l'époque tibérienne comporte un plat à paroi oblique et des lèvres du service Ia.

A. D.

\section{LA SIGILLÉE}

\section{LA SIGILLÉE DE LA MUETTE}

\section{CHRONOLOGIE DES PRODUCTIONS}

Les hypothèses d'origine (15 avant - 15 après J.-C.) sont fondées sur les comparaisons de faciès céramiques que l'on pouvait faire à l'époque : le mobilier de trois sites majeurs du limes, à savoir Dangstetten, Rödgen et Haltern, n'avait pas fait l'objet de publications détaillées, la datation proposée reposait donc essentiellement sur des observations générales faites d'après un examen rapide de l'ensemble du matériel, et notamment sur la présence et l'abondance relative de formes du service II attestées à Haltern entre 7 avant et 9 après J.-C.

L'analyse du mobilier des principaux contextes mis au jour sur l'atelier permet aujourd'hui de distinguer très nettement deux périodes ou "phases" de production. Le mobilier de la période 1 se caractérise par un très faible degré de standardisation tandis que celui de la période 2 s'apparente davantage aux horizons du limes, et en particulier à l'horizon Haltern. Les éléments et les indices qui nous ont amenés à proposer une chronologie pour ces deux phases d'activité ont été décrits et expliqués plus haut de façon détaillée. Aussi nous contenterons-nous, ici, de rappeler l'essentiel des informations recueillies.

Les principaux indices de datation ne sont pas tous au même niveau, ni de même valeur : ils sont d'une part d'ordre statistique, d'autre part d'ordre typologique.

Les très faibles pourcentages de service II par rapport au service I dans tous les ensembles concernés de la période 1 indiquent une datation beaucoup plus ancienne que celle des camps de Rödgen, Oberaden et, a fortiori, de Haltern. Le seul site sur lequel le service I l'emporte de très loin sur le service II est Dangstetten dont la durée d'occupation couvre les années 15/9 avant
J.-C. Parallèlement, la très nette prépondérance du service IB sur le service IC semble aller dans le même sens.

On a donc tout lieu de supposer que la première phase d'activité de la Muette s'inscrit dans un horizon chronologique précoce, correspondant ou légèrement antérieur à celui de Dangstetten.

Le déséquilibre numérique entre les services I ct II persiste dans les contextes qui définissent la période 2 . En termes statistiques. l'évolution du mobilier se traduit plus clairement par une très nette augmentation du service IC par rapport au service IB.

D'une manière générale, on observe, dans la période 1, un très faible degré de standardisation, tout à fait surprenant si l'on suppose, comme on le faisait jisqu'alors, que l'atelier a fabriqué et diffusé massivement ses produits tout au long de son activité. Les formes d'assiettes et de bols du service II de la période 1 ne sont quasiment pas attestées sur le limes, et, de surcroît, totalement inconnues sur les sites d'habitats, qu'ils soient lyonnais, régionaux ou autres et qu'il s'agisse de camps militaires ou de sites d'habitats. La remarque vaut également pour les assiettes du service IC dont l'hétérogénéité contraste singulièrement avec le très fort degré de standardisation que montre le matériel de Haltern et que l'on pouvait même déjà constater dans l'horizon plus ancien de Dangstetten.

Le mobilier de l'ensemble II montre des changements qui traduisent clairement une évolution chronologique : on assiste en effet à la disparition des types rares ou inédits qui caractérisaient la sigillée de l'ensemble I. Le service II se diversifie et les formes de plats, d'assiettes ou de bols attestées dans les différents contextes se rapprochent dawantage des formes classiques extrêmement abondantes sur le limes, en particulier à Haltern. Parallèlement, le service IC, qui comprenait auparavant un grand nombre de variantes pratiquement inconnues sur les sites de référence, se normalise de façon évidente jusqu’à offrir une image quasi identique à celle donnée par le matériel de Haltern.

Cela dit, les productions sigillées de la période 1 , logiquement destinées à la commercialisation, posent le problème de leur extrême rareté sur les sites de consommation aussi bien lyonnais qu'extérieurs. Cette absence peut s'expliquer par une courte durée de cette première phase, correspondant à une période d'essai ou de mise en train de la production. Il convient de signaler que cette première phase utilise majoritairement des pâtes très calcaires qui seront délaissées par la suite pour des argiles 
dont les pourcentages de chaux varient autour de $12 \%$, comme les sigillées arétines.

Ce phénomène est-il imputable à leur chronologie précoce, c'est-à-dire tout simplement au fait que nous ne connaissons pas à ce jour de contextes strictement contemporains? L'abondance relative sur les sites de référence de parois fines lisses et décorées semblables à celles produites à Lyon au cours de la période 1 tend à fragiliser cette direction de recherche et laisse entrevoir la complexité de cette question. La deuxième phase de production est un peu mieux représentée sur les sites de consommation mais la grande période de diffusion de la sigillée lyonnaise ne correspond pas aux céramiques livrées par les fouilles de la Muette. Il est possible que l'absence dans l'atelier de matériel correspondant à la grande période de diffusion soit due au hasard de la fouille, mais il n'est pas exclu non plus que celle-ci provienne d'un autre site atelier, installé en un autre secteur du val de Saône ou ailleurs dans Lyon. Il semble évident que les céramiques sigillées récoltées lors de la fouille de la Muette ne représentent qu'une partie des productions de l'atelier et des sigillées lyonnaises. Cela donne sans aucun doute une vision tronquée et déformée de la production des ateliers lyonnais. La découverte, en aval du site de la Muette, de dépotoirs d'ateliers de sigillée, indique que la zone de production s'étendait sur une surface importante. De même, la découverte d'un moule de sigillée dans les fouilles de Saint-Vincent pourrait être l'indice d'une extension des ateliers encore plus loin en aval. Ces témoins peuvent aussi être l'indice d'un déplacement dans le temps des zones de productions, le long des rives de la Saône.

M. G.

\section{L'ORGANISATION INTERNE DU TRAVAIL ET LE PROBLÈME DE LA COMMERCIALISATION}

L'étude des timbres sigillés qui complète l'analyse typologique révèle d'autres phénomènes qui tendent à valider l'hypothèse de deux périodes de production. Bien que globalement majoritaires dans les deux ensembles, les quatre marques principales (Attivs, Rasinivs, Sentivs, Thyrsvs) sont représentées de l'un à l'autre par des graphies différentes, de surcroît utilisées pour des formes elles aussi différentes. On observe également, en ce qui concerne les autres marques, de fortes discordances numériques, voire la disparition ou l'apparition de telle ou telle signature d'une période à l'autre.

L'analyse fine de ces timbres permet ensuite et surtout d'en finir avec la notion de service qui, bien que toujours valide à plusieurs égards, induit une vision simpliste de la production des vases sigillés et, partant, de leur diffusion. Elle conforte les hypothèses naguère émises par $\mathrm{S}$. von Schnurbein sur la spécialisation des potiers et sur les problèmes de commercialisation liés à cette réalité. En fait, on s'aperçoit que seuls les quatre potiers les plus représentés en nombre ont fabriqué, semble-t-il indifféremment, toutes les formes et tous les types de vases; parallèlement, la plupart des autres potiers se sont spécialisés dans la fabrication d'une forme ou d'un type, voire dans quelques cas, d'une variante précise d'un type donné. Les déséquilibres constants entre les bols et les assiettes des services IB, IC et du service II, qu'ils portent ou non les mêmes signatures, montrent bien par ailleurs que la volonté d'élaborer des services de vaisselle à proprement parler n'existait tout simplement pas. De telles circonstances semblent indiquer que les potiers fixaient ensemble un programme de production en se répartissant le travail selon leurs capacités matérielles (petites ou grandes officines) et qu'ils devaient ensuite s'associer au stade de la cuisson afin d'obtenir des fournées permettant de procéder à des assortiments de différentes formes. Toute la question reste alors de savoir qui décidait de ces assortiments, c'est-à-dire à quelle demande l'offre s'adaptait-elle? Il est probable que les potiers étaient relayés par d'autres instances à cette dernière étape du travail (marchands, grossistes, transporteurs?), instances dont aucun témoignage épigraphique ou autre n'a, à ce jour, prouvé la réalité.

M. G.

\section{LA SIGILLÉE VIENNOISE}

Un des résultats nouveaux concernant la sigillée est la mise en évidence d'une production viennoise. Celle-ci a été démontrée par les analyses, qui ont confirmé l'attribution à Vienne de l'estampille MENA/AVILI (Desbat, Picon, 1992), mais notre connaissance de ce nouvel atelier est plus que limitée. À Vienne même, les quelques tessons autribuables à cet atelier ont été mis au jour dans des contextes du tout début de notre ère. Cela n'est pas suffisant pour dater la phase d'activité de l'atelier. Il serait pourtant très important de savoir si l'installation de cet 
atelier a précédé ou non celle de la Muette. Cette découverte souleve une fois encore la question des rapports entre les ateliers lyomnais et viennois; elle pose également en d'autres termes la question de l'atelier d'Ateivs.

\section{LA QUESTION D'ATEIVS}

Un des résultats majeurs des analyses a été la mise en évidence d'un atelier gaulois d'Ateirs (Picon, Garmier, 1974) ayant largement diffusé ses productions sur le limes rhénan, comme l'ont confirmé par la suite les analyses effectuées sur le matériel de Haltern (von Schnurbein, 1982).

Les premiers travaux ont conclu que cet atelier devait être lyonnais compte tenu de la composition des pâtes, proche de celle de la Muette. Paradoxalement, les foulles de la Muette n'ont pas livré de production d'Ateivs. Il faut donc supposer soit que la production d'Ateivs appartient aux phases récentes de l'atclier qui n'ont pas été mises en évidence par les foulles, soit que l'atelier se trouvait sur un site voisin.

Toutefois, les avancées réalisées dans les études de laboratoire depuis la publication des premiers résultats, en particulier dans la séparation des ateliers lyonnais et viennois, nécessiteraient de reprendre la question d'Ateivs arec de nouvelles séries d'analyses.

Il faudrait être certain qu'une partie des productions d'Ateivs ne puisse être attribuée à Vienne, hypothèse qui arait déjà été envisagée par $\mathrm{M}$. Picon. On ne peut exclure, dans l'état actuel de nos connaissances, l'hypothèse selon laquelle l'atelier d'Ateivs se serait installé dans un premier temps à Vienne, avant d'être transféré à Lyon, ni que cet atelier soit finalement viennois.

A. D.

\section{LES CÉRAMIQUES À PAROIS FINES}

Les céramiques à parois fines représentent une part très importante de la production des ateliers lyonnais précoces. Les types produits dans les ateliers de Loyasse et de la Muette sont variés et l'on compte une douzaine de types, sans les gobelets d'Aco. Si l'on excepte les gobelets cylindriques à pied mouluré (Mayet, 1975, forme XII), le répertoire des ateliers diffère. Cependant le peu de matériel recueilli à Loyasse ne nous donne qu'un aperçu de la production de cet atelier, qui a pu être plus diversifiée.
On doit pourtant souligner l'absence, à Loyasse comme à la Muette, de types bien présents dans les contextes augustéens régionaux, mais aussi sur les sites de consommation contemporains.

Il s'agit en premier lieu des " Rippenbecher » (Mayet, 1975, forme III B). Leur absence à la Muette peut s'expliquer pour des raisons chronologiques et nous pourrions admettre que cette forme n'est plus produite à l'époque de la Muette. Elle est en revanche bien attestée dans le camp de Dangstetten (avec 15,8\% des céramiques à parois fines) où elle représente le deuxième type après les gobelets d'Aco (Fingerlin, 1986). On la trouve aussi à Saint-Romain-en-Gal et sur les sites lyonnais de la rue des Farges, du Verbe-Incarné ou du "sanctuaire de Cybèle". Son absence à Loyasse peut être mise sur le compte de la faiblesse de notre échantillon. On peut encore envisager qu'il s'agit d'une production italique qui n'a pas fait l'objet de copies gauloises. Pourtant cette hypothèse paraît contredite par l'absence de Rippenbecher aussi bien au Magdalensberg (Schindler, Kaudelka, 1975) qu'en Espagne (Mayet, 1975; Lopez Mullor, 1986). Il est donc probable qu'il s'agisse bien d'une production gauloise, mais dont les ateliers restent à découvrir. La même question se pose pour deux autres types:

- d'une part, les gobelets à lèvre concave à décor de gros picots (Mayet forme III B) dont les pâtes paraissent identiques à celles des Rippenbecher ;

- d'autre part, les gobelets sphériques à panse striée et lèvre érasée (Haltern 43). Ces derniers sont bien représentés à Dangstetten où ils arrivent en troisième position avec $11 \%$.

C. Grataloup (1988) avait attribué ce type de gobelet à la Muette, mais l'étude détaillée du matériel de l'atelier n'a pas apporté de confirmation. Seuls deux fragments ont été recueillis dans les fouilles de l'atelier et le centre de production est à rechercher ailleurs.

A. D.

\section{CHRONOLOGIE DES VASES À PAROIS FINES LISSES}

Les gobelets cylindriques et les gobelets tronconiques représentent les deux seuls types fabriqués à la fois à Loyasse et à la Muette. Ils composent l'essentiel des rases à parois fines lisses de Loyasse, mais apparaissent ensuite exclusivement dans les productions de la première phase d'activité de la Muette. De telles circonstances leur confè- 
rent d'emblée un caractère ancien que viennent d'ailleurs confirmer les comparaisons numériques effectuées d'après différents horizons lyonnais et viennois auxquels s'ajoute l'horizon Dangstetten : ces deux types sont totalement absents des premiers habitats du VerbeIncarné (40 avant J.-C.), relativement bien représentés dans les contextes datables de 30/10 avant J.-C. (Lyon/Loyasse L3, SRG 1, Dangstetten) pour disparaître assez rapidement par la suite, comme le montre l'horizon classique du Verbe-Incarné (V.I. 2), (tabl. XXI). On peut donc supposer, au vu de ces données, que les gobelets cylindriques et les gobelets tronconiques ont eu une durée de vie très courte, vraisemblablement comprise dans les années $30 / 10$ avant J.-C.

Les gobelets-tonneaux et les bols hémisphériques sont inscrits au seul répertoire de la Muette. Leur répartition numérique de l'ensemble I à l'ensemble II nous apporte d'autres renseignements d'ordre chronologique. On observe en effet que les gobelets-tonneaux passent de $58 \%$ des vases lisses dans la période 1 à $15 \%$ dans la période 2 ; tout se passe comme s'ils cédaient le pas aux bols hémisphériques qui voient, quant à eux, leurs effectifs passer de 7 à $80 \%$ des bords. Le mobilier des sites de consommation illustre assez bien ce changement de situation (tabl. XXI) : les gobelets-tonneaux apparaissent timidement (2 exemplaires sur 169 vases) à Lyon dans l'horizon Loyasse L.3 (30/20 avant J.-C.) et se retrouvent essentiellement dans le mobilier de SRG 1 (vers 20 avant J.-C.) et de Dangstetten (15/9 avant J.-C.) pour se réduire enfin à $7 \%$ au début du $\mathrm{I}^{\mathrm{cr}} \mathrm{s}$. Parallèlement, les bols hémisphériques n'interviennent qu'à partir de l'horizon Dangstetten pour devenir très rapidement la forme lisse la plus courante dans les contextes augustéens classiques (fig. 91).

Aux côtés de ces quatre types très largement majoritaires figurent dans l'un et l'autre atelier des séries apparemment inédites. Il s'agit pour Loyasse de vases à décors guillochés, pour la Muette de gobelets à pâte siliceuse (groupe des "bords striés") et de vases à pâte calcaire reprenant le plus souvent les profils des types produits en pâte siliceuse. Ces formes ne sont pas plus attestées à Lyon que sur d'autres sites de consommation, locaux, régionaux ou autres, et leur présence dans les deux ateliers pourrait alors seulement rendre compte d'essais ou de tentatives vite abandonnés. On note cependant que les gobelets à bord strié et les vases à pâte calcaire de la Muette figurent essentiellement dans le mobilier de la période 1 aux côtés de ces productions de sigillée dont la destination pose des problèmes; l'absence de parallèles pour ces séries isolées et très limitées en nombre pourraitelle alors être due, au moins en partie, à la rareté de contextes strictement contemporains de cette première phase de production?

M. G.

\section{LES GOBELETS D'ACO}

L'association des frises, des signatures et des décors permet de distinguer aisément la production de l'atelier de Loyasse de celle de la Muette, et de différencier les gobelets des potiers ayant travaillé à la Muette, même sur des fragments non signés. En revanche, la très grande similitude entre les productions de Vienne (SaintRomain-en-Gal) et de la Muette, issues pour une grande part des mêmes moules, rend très difficile, pour ne pas dire impossible, l'attribution, à l'un ou l'autre des deux ateliers, des fragments de gobelets d'Hilarvs ou de Chrysippvs.

Sur les deux sites, il est peu probable que nous connaissions la totalité de la production. L'absence, dans la fosse de Saint-Romain-en-Gal, de gobelets signés Philarcvrvs, Philocrates ou T. Cavivs ne signifie pas pour autant que ces décorateurs n'aient pas travaillé à Vienne. On doit remarquer en particulier que des gobelets de $T$. Cavivs se rencontrent dans les niveaux d'habitat voisins de l'atelier.

Les ensembles stratifiés fournis par les sites lyonnais et viennois ont permis de préciser la chronologie des gobelets d'Aco. À Vienne, les horizons chronologiques de la

\begin{tabular}{|l|l|c|c|c|c|c|}
\hline \multicolumn{1}{|c|}{ ATELIERS } & \multicolumn{1}{|c|}{ TYPES } & $\begin{array}{c}\text { Verbe- } \\
\text { Incarné 1 }\end{array}$ & Loyasse L3 & SRG 1 & Dangstetten & $\begin{array}{c}\text { Verbe- } \\
\text { Incarné 2 }\end{array}$ \\
\hline Loyasse et Muette & gobelets cylindriques & 0 & 51 & 7 & 3 & 1 \\
\hline Loyasse et Muette & gobelets tronconiques & 0 & 0,6 & 2,8 & 11,4 & 3,6 \\
\hline Muette & gobelets-tonneaux & 0 & 1,2 & 18,3 & 16,5 & 7,3 \\
\hline Muette & bols hémisphériques & 0 & 0 & 0 & 3,6 & 74,5 \\
\hline
\end{tabular}

Tabl. XXI. Pourcentages des principaux types de vases à parois fines lisses sur. differents sites augustéens. 


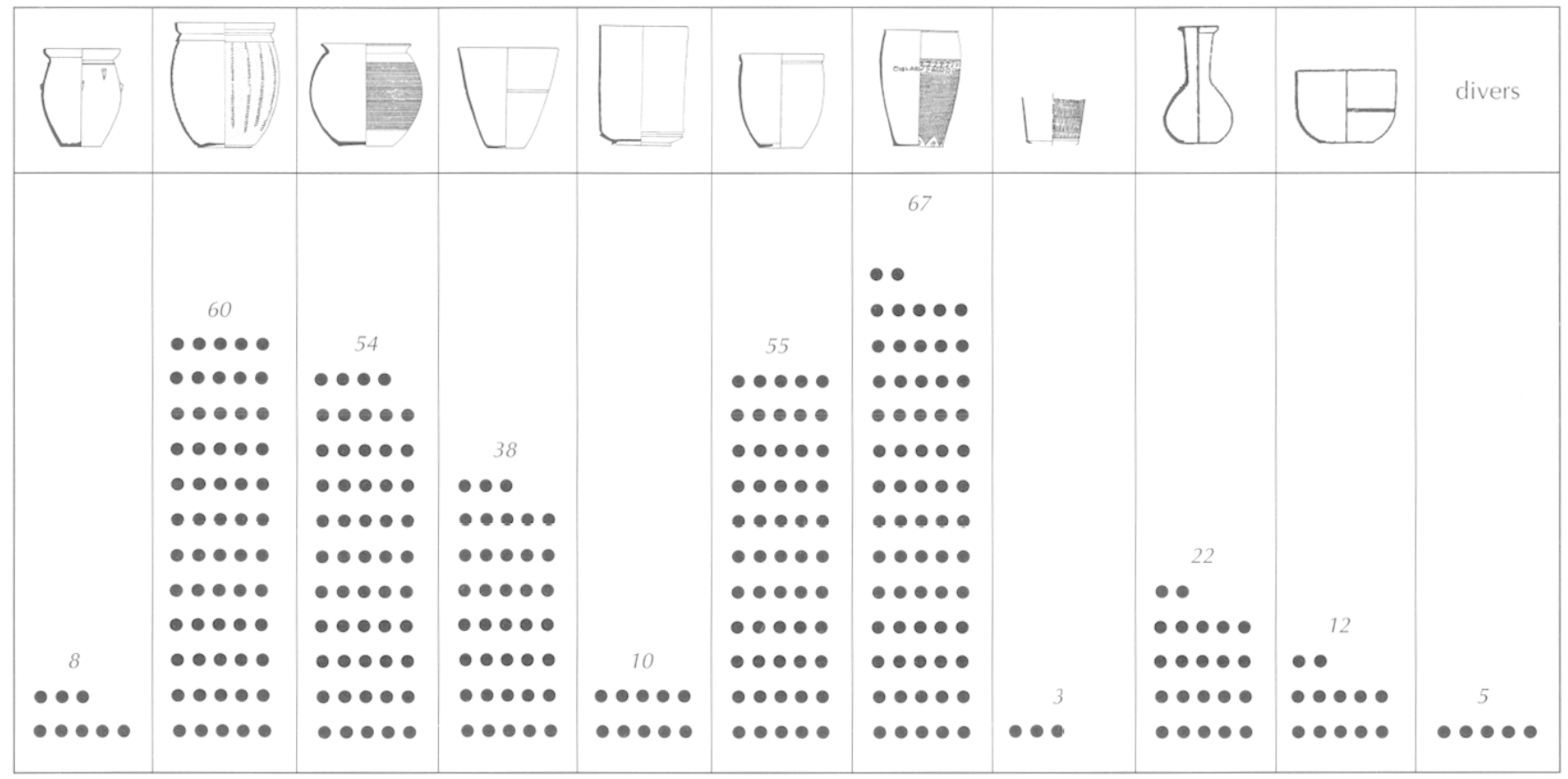

Fig. 91. Répartition des gobelets de Dangstetten par types (calcul du NMI effectué sur les bords uniquement).

maison des Dieux Océan montrent que les gobelets d'Aco sont majoritaires dans la première phase, où ils représentent $52 \%$ des céramiques à parois fines (Desbat et alii, 1994, p. 74, tabl. 8 et 9). Un pourcentage aussi élevé peut s'expliquer par la présence d'un atelier voisin (Desbat, 1985) mais il reste que le nombre de gobelets diminue dans les phases suivantes. À Lyon, les nouveaux contextes fournis par les fouilles conduites à l'emplacement du prétendu sanctuaire de Cybèle, ainsi que ceux correspondant à la première trame urbaine sur le site du VerbeIncarné, attestent leur présence durant la période 40-20 avant J.-C. (Desbat et alii, 1989). Une partie de ces gobelets est d'origine indéterminée et une provenance italique est possible, bien que non démontrée. Le reste provient de l'atelier de Loyasse, ce qui confirme l'ancienneté de l'atelier et la date proposée.

Dans les contextes plus récents, où les gobelets d'Aco sont encore présents, les productions de Loyasse disparaissent au profit de celles de Saint-Romain-en-Gal et de la Muette. I a similitude des décors rend malaisée la distinction entre les deux ateliers, qui présentent très certainement un décalage chronologique. L'antériorité de l'atelier de Saint-Romain-en-Gal par rapport à celui de la Muette est étayée par plusieurs indices. Le premier est la production de gobelets à glaçure plombifère à SaintRomain-en-Gal comme à Loyasse, alors que la Muette a abandonné ce type de production. Le deuxième, plus déterminant, est l'utilisation à Lyon de moules de composition viennoise. On doit donc supposer le transfert de l'atelier de Vienne à Lyon. La durée de vie de l'atelier de Saint-Romain-en-Gal est difficile à fixer. La phase de production mise en évidence dans les fouilles de la maison des Dieux Océan (Desbat, 1985) correspond à une période très courte, la fosse de ratés de cuisson pouvant être le résultat d'une seule cuisson!

Les couches d'occupation des habitats voisins montrent la présence des gobelets sur une durée de plusieurs années, qui couvrent les horizons I et II mais il reste la possibilité qu'une part importante du matériel soit résiduelle en SRG II (Desbat et alii, 1994).

À Lyon, la grande quantité de gobelets livrée par la fouille de la Muette suggère une production plus importante et de plus longue durée, mais cette supposition doit être nuancée. La production de gobelets d'Aco ne représente en effet que $3,7 \%$ des céramiques à parois fines, et celle-ci a été très limitée dans le temps. Il faut noter en premier lieu l'absence de gobelets dans l'ensemble II correspondant à une phase de production plus récente. La 
production de gobelets d'Aco à la Muette paraît donc limitée à la première phase, antérieure à la grande diffusion des sigillécs. Unc confirmation est donnée par la faible quantité de gobelets d'Aco livrée par les fouilles de Haltern, d'Oberaden, et par les ensembles lyonnais contemporains, en particulier au Verbe-Incarné (Genin, 1993). Les gobelets d'Aco de Chrysippss, Hilarvs, Philarcvrvs, T. Cavivs avec les mêmes décors qu'à la Muette sont davantage présents à Dangstetten (Fingerlin, 1986) où ils représentent $24 \%$ des céramiques à parois fines, mais il reste à démontrer qu'il s'agit bien de produits de Lyon et non de Saint-Romain-en-Gal. Le traitement des fonds tendrait à prouver que la majorité des gobelets attribućs à la Muette sur différents sites du limes rhénan proviendraient plutôt de Saint-Romain-en-Gal. Tout au moins, ceux-ci ne peuvent appartenir à la production correspondant aux vases recueillis dans l'atelier de la Muette.

A. D.

\section{LES RELATIONS ENTRE LES ATELIERS DE LYON ET DE VIENNE}

La découverte des productions augustéennes viennoises a mis en lumière des relations étroites, voire certaines filiations, entre les ateliers lyonnais et viennois. La mise en évidence d'une production de sigillée à Vienne, ainsi que de gobelets d'Aco, montre que ces deux villes ont une production abondante et diversifiée, comprenant aussi bien céramiques fines que céramiques communes, y compris culinaires, et amphores. Ces deux centres urbains paraissent donc avoir produit, au début de l'Empire, toute la gamme des céramiques pour la consommation locale, mais aussi pour l'exportation. On doit cependant noter que ces deux centres de production ne semblent pas avoir eu la même importance. Le nombre d'ateliers découverts à Lyon, mais aussi les données que nous possédons sur la diffusion des produits, permettent de supposer que la production de sigillée, de céramiques à parois fines ou d'amphores à Vienne a été bien moindre et que Lyon a constitué le principal centre de production. La découverte à Saint-Romain-en-Gal de vestiges d'une production de gobelets d'Aco de Chrysipprs et d'Hilarvs Aco avec les mêmes décors qu'à la Muette illustre le rapport entre ces deux centres. Il apparaît que les mêmes moules ont été utilisés à Saint-Romain-
en-Gal et à la Muette et qu'il y a donc eu transfert de matériel de l'un à l'autre et même déplacement de l'atelier. Certains indices comme les données chronologiques, ou encore la composition de plusieurs des moules trouvés dans l'atelier de la Muette, permettent de conclure que l'atelier d'abord installé à Vienne s'est déplacé ensuite à Lyon. Il est difficile en revanche d'estimer la durée de fonctionnement de l'atelier à Vienne.

On comprend moins les relations pouvant exister entre l'atelier de Loyasse et celui de Saint-Romain-en-Gal. Pourtant, on constate la fabrication sur ces deux sites de gobelets signés Hilarvs Aco. La production de ces deux ateliers reste toutefois très différenciée et les décors comme les signatures se distinguent aisément de l'un à l'autre. La chronologie établie pour ces sites fait de Loyasse l'atelier le plus ancien, dont la durée de fonctionnement reste hypothétique (entre 30 et 15 avant J.-C.). Il faudrait donc admettre soit que l'atelier de gobelets d'Aco de Saint-Romain-en-Gal ait succédé à celui de Loyasse (à moins de supposer une phase de production plus ancienne, encore inconnue, à Saint-Romain-en-Gal), soit que les périodes d'activité des deux ateliers se soient superposées, avant que l'atelier de Saint-Romain-en-Gal ne soit transféré à Lyon.

On peut émettre l'hypothèse que ce déplacement est dû à l'installation ou à l'essor de l'atclier de la Muette. Le regroupement de l'atclier de gobelets d'Aco avec l'atelier de sigillée peut s'expliquer soit par la volonté des negotiatores d'avoir à Lyon ces deux produits pour les diffuser ensemble, soit par celle des potiers ou d'autres négociants voyant dans l'installation d'une succursale d'Arezzo, la possibilité de débouchés pour leur production. Cela dit, on doit constater le regroupement sur le même site de production d'une succursale d'Arezzo et d'un atelier qui procède d'une autre origine.

A. D.

\section{ATELIERS GAULOIS OU SUCCURSALES ITALIQUES?}

Lorsqu'ont été découverts les premiers atcliers lyonnais, la tendance a été d'opposer les ateliers producteurs d'imitations de sigillée comme Loyasse à l'atelier de la Muette, succursale d'Arezzo et de considérer les premiers comme des ateliers de tradition indigène ayant assimilé les techniques de tournage mais non les procédés de cuis- 
son permettant la réalisation du vernis. Il semble maintenant évident qu'il faille reconsidérer cette perception. L'atelier de Loyasse comme celui de Saint-Romain-en-Cal peuvent être considérés comme des ateliers de tradition italique. Le choix des argiles calcaires pour les imitations de sigillée les distingue des ateliers imitant le répertoire italique avec des techniques indigènes (terra nigra et terra rubra par exemple).

De même, la production de céramiques à parois fines, de gobelets d'Aco et de céramiques à glaçure plombifère attestent qu'ils n'ont rien d'indigène. La présence de tria nomina parmi les rares estampilles connues va dans le même sens. La nature des productions associées aux imitations de sigillée, comme les gobelets d'Aco ou les céramiques à glaçure plombifère, suggère des liens avec la vallée du Pô, et il est possible que des ateliers comme ceux de Loyasse ou Saint-Romain-en-Gal soient eux-mêmes des succursales italiques au même titre que la Muette, mais des succursales d'ateliers padans, ou sans être des succursales au sens strict, qu'ils aient accueilli des potiers venant de cette région. Bien que les gobelets d'Aco produits à Lyon et à Vienne se distinguent par leur décor et leurs signatures (Vegas, 1969-1970) des productions padanes connues, c'est bien dans cette région où l'on connaît de nombreux ateliers ayant fabriqué ces gobelets que l'on doit rechercher l'origine des ateliers lyonnais et viennois. Il convient de noter, bien qu'une homonymie soit toujours possible, que le nom d'Hilarvs se rencontre sur quelques gobelets du Magdalensberg (Schindler, Kaudelka, 1980) et qu'un gobelet de la nécropole d'Ottobiano comporte la signature HIL.ARVS. CAVI. S (ervus) $\mathrm{F}$ : gobelet de la tombe 36 d'Ottobiano conservé au musée Iomellino (Vannaci-Lunazzi, 1987). Peut-être faut-il voir dans le Cavivs dont Hilarvs est l'esclave le $T$. Cavivs qui est venu s'installer à Lyon. On connaît en outre un gobelet signé PHII ARCVRVS CAVIVS à Montegrotto, ce qui paraît confirmer les relations entre Lyon et la vallée du Pô.

\section{LA DIFFUSION DE LA PRODUCTION DES ATELIERS LYONNAIS}

S. von Schnurbein (Conspectus, 1990) et C. M. Wells (1992) ont classé les ateliers de sigillée gaulois en trois catégories :

1. les ateliers à l'activité de longue durée et de grande diffusion ;
2. les ateliers à l'activité de courte durée mais avec une grande diffusion ;

3. les ateliers à l'activité de courte durée, avec une diffusion seulement locale ou régionale.

La deuxième catégorie n'est représentée que par l'atelier de la Muette et la grande question concernant cet atelier est la raison de son déclin rapide : pourquoi a-t-il connu une durée de vie si courte? Je reviendrai sur cette question.

L'atelier de Loyasse est classé quant à lui dans la troisième catégorie avec des ateliers comme Bram, mais cette classification demande à être révisée.

\section{L'ATELIER DE LOYASSE}

La découverte très ponctuelle de résidus d'ateliers céramiques dans le cimetière de Loyasse ne permet de se faire qu'une idée très imparfaite de cet atelier et a minimisé son importance. Pourtant certains indices laissent supposer une diffusion qui dépasse le cadre régional. Les décors très particuliers des gobelets d'Aco de Loyasse permettent de les distinguer de ceux de la Muette et de SaintRomain-en-Gal et d'esquisser une carte de distribution (fig. 92). La rareté des sites ayant livré des vestiges antérieurs à 20 avant J.-C. limite les possibilités et seuls huit sites, en dehors de Lyon et de Vienne, ont fourni de tels gobelets :

- Paris :

- rue Cujas : Bouthier, 1984, fig. 30b, frise $n^{\circ} 8$ VS ACO (lu à tort ACAVSTVS ACO).

- rue Pierre-et-Marie-Curie : Bouethiez, 1993, fig. 1, 6, frise $n^{\circ} 13$; fig. 1,6 , frise $n^{\circ} 2$.

- Bavai : Biévelet, 1969, fig. 2, frise $n^{\circ} 2$.

- Bâle: Fellmann, 1955, pl. 18, 4, frise ${ }^{\circ} 9$; FürgerGunti, 1979, fig. 15, 1 et pl. 6,1, frise $n^{\circ} 9$ avec ACO et dauphin ; fig. 15, 3, frise ${ }^{\circ} 9$; Fürger-Gunti, 1979, pl. 24, 414 , frise $n^{\circ} 9$; Helmig, Jaggy, 1990, fig. 10, 16, frise $n^{\circ} 2$ ARVS ACO; fig. 10, 18, frise $n^{\circ} 3$; fig. 10, 20, frise $n^{\circ} 5$.

- Zürich: Vogt, 1948, pl. 34, 6, frise $\mathrm{n}^{\circ} 1$ avec dauphin.

- Goeblingen-Nospelt : Metzler, 1984 tombe B, p. 296, $n^{\circ} 147$, frise $n^{\circ} 9$ HII ARVS ACO entre deux dauphins.

- Neuss : Vegas, 1975 , pl. 5, 1, frise $n^{\circ} 9$; pl. 5, 4, frise $\mathrm{n}^{\circ} 1$; pl. 5,5 , frise $\mathrm{n}^{\circ} 2$.

- Liberchies: Graff, 1975, pl. 7, 2, frise $n^{\circ} 1$; Claes, Milliau, 1964, fig. 13, frise $n^{\circ} 7$; Claes, 1969, fig. 25, même gobelet. Le même fragment a été repris par Vanderhoeven, 1989, fig. 10,15 , frise $\mathrm{n}^{\circ} 7$. 


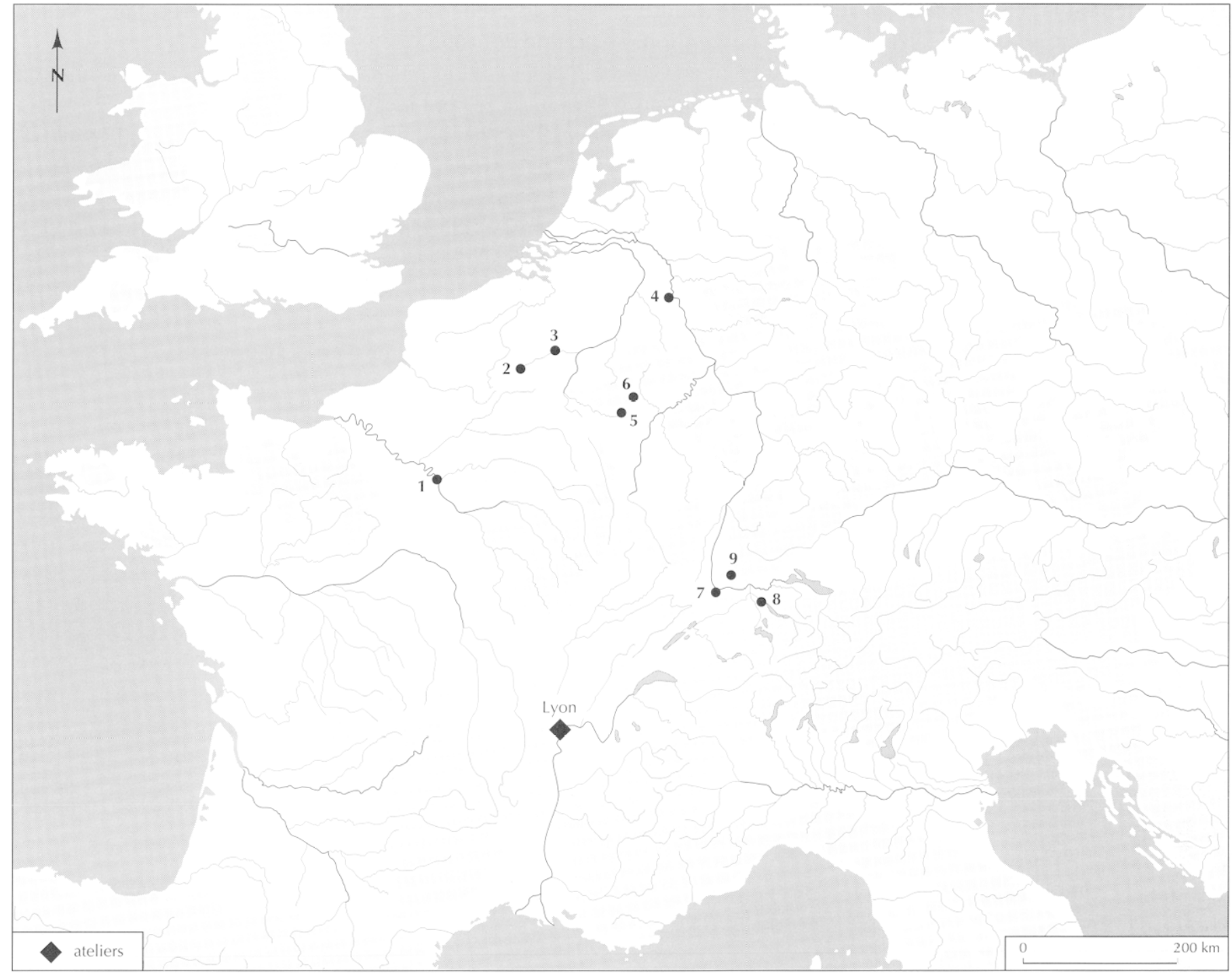

Fig. 92. Cante de répartition des gobelets d'Aco de Loyasse : 1, Paris; 2, Bavai ; 3, Liberdies; 4, Neuss; 5, Titelberg; 6, Goeblingen-Nospelt; 7, Bâle; 8, Zürich; 9, Dangstetten.

- Dangstetten : Fingerlin, 1986, pl. 25, 543, 16, frise $n^{\circ} 1$, avec dauphin.

- Titelberg: Metzler, 1995, p. 525, fig. 266, 7 , frise $n^{\circ} 8$; fig. 266, 14 , frise $n^{\circ} 9$.

Il ne fait pas de doute qu'une enquête plus systématique permettrait d'étoffer cette carte. La présence sur de nombreux sites de céramiques précoces à vernis noir ou rouge, pour lesquelles l'origine lyomnaise est probable, irait dans le même sens. Il reste toutefois à déterminer ce qui peut être la part de Loyasse par rapport à d'autres ateliers lyonnais ou rhodaniens contemporains. La mise en évidence, par les analyses, de productions identiques à celles de Loyasse, mais issues d'autres ateliers, complique les choses mais montre que, sinon Loyasse même, d'autres ateliers lyonnais du même type ont eu une diffusion plus large qu'on ne l'imaginait. L'attribution à Lyon de céramiques " campaniennes " trouvées en Suisse (Kacnel, Magetti, 1986) prouve que, contrairement à l'idée qui a prévalu jusqu'ici, les ateliers lyonnais antéricurs à la Muette connaissaient déjà une assez large diffusion. 


\section{L'ATELIER DE LA MUETTE}

La diffusion de l'atelier de la Muette paraît plus facile à établir, non seulement parce que les productions de l'atelier sont mieux connues que celles de Loyasse mais encore parce qu'il existe un plus grand nombre de sites avec une occupation contemporaine de la grande phase de diffusion de l'atelier. L'analyse du matériel de Haltern a permis en outre de compléter notre connaissance des productions de sigillée en attribuant à l'atelier de nouvelles estampilles, mais aussi en permettant de quantifier la part de la Muette dans le matériel de Haltern, où elle représente $50 \%$ de la sigillée.

On peut actuellement dresser une carte de distribution des céramiques sigillées de la Muette qui montre une large diffusion (fig. 93). Celle-ci n'est pas seulement tournée vers la vallée du Rhin et les camps militaires mais concerne aussi bien des sites civils dans le nord de la Gaule, le val de Loire et la Bretagne. Ainsi à Bavai, la sigillée de la Muette représenterait près de $30 \%$, selon $\mathrm{M}$. Vanderhoeven (1989) et Tongres fournirait un pourcentage comparable.

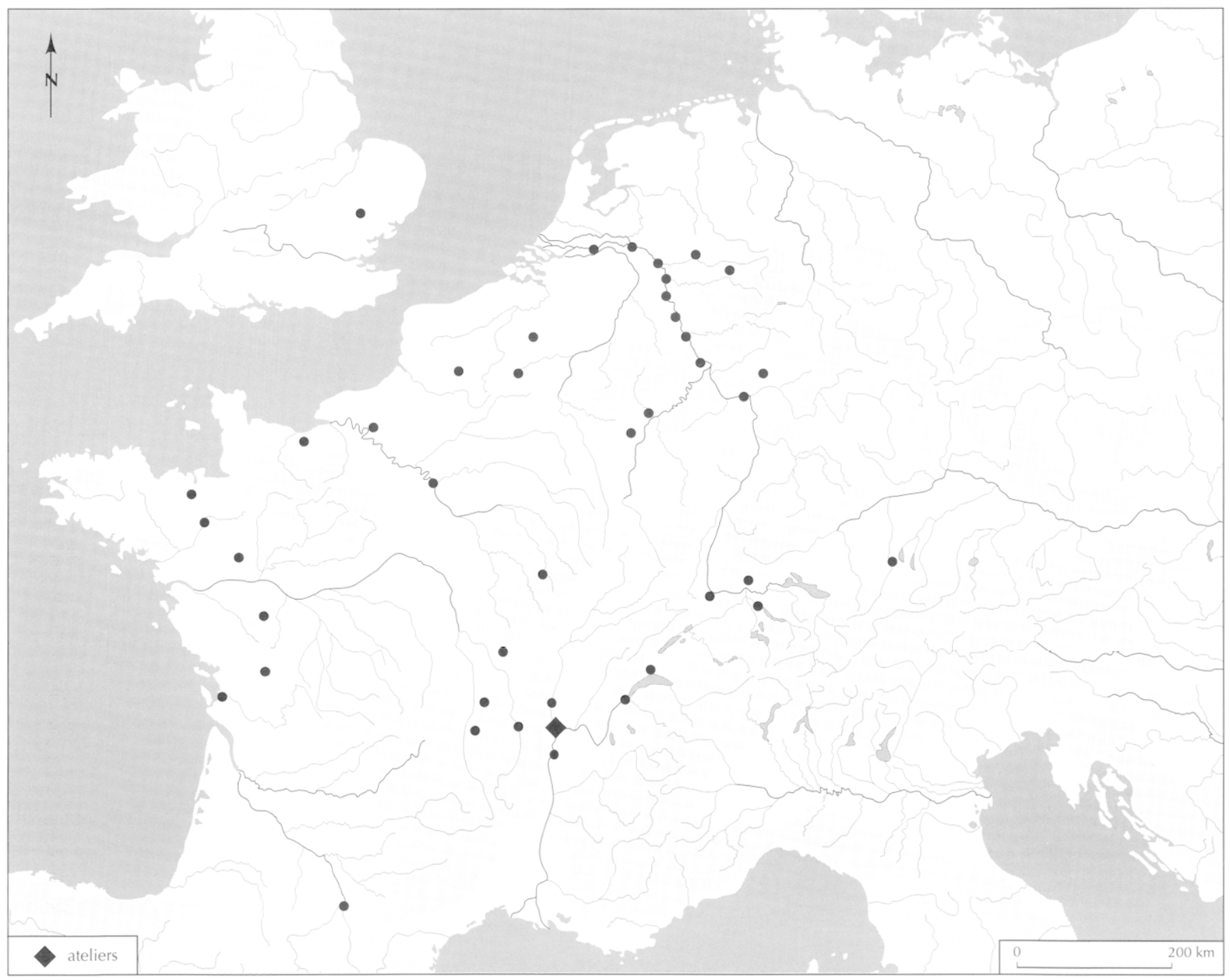

Fig. 93. Carte de répartition des productions de la Muette, sigillée et gobelets d'Aco. 
Les cartes de répartition des gobelets d'Aco signés Hilarvs Aco, Chrysippvs ou T. Cavivs et Philarcvrvs (fig. 94 et 95) montrent éyalement une diffusion assez large qui recoupe celle des sigillées. Il faut néanmoins rappeler que la similitude entre les décors des gobelets de la Muette et ceux de Saint-Romain-en-Gal rend toujours possible une confusion entre ces deux ateliers et il n'est pas certain que tous ces gobelets proviennent de Lyon. Leurs caractéristiques techniques, en particulier le traitement des pieds, laisseraient plutôt supposer qu'une grande partie d'entre eux proviendrait de Vienne. Mais il faudrait être certain que ce critère soit déterminant. Le problème se pose différemment pour la sigillée, mais le risque d'exagérer l'importance de la Muette, en lui attribuant les productions d'autres ateliers régionaux, existe néanmoins. Il faut noter en premier lieu que les pourcentages obtenus sur des sites comme Bavai ou Tongres sont le résultat d'un classement visuel des pâtes et des vernis, reprenant celui de Haltern (von Schnurbein, 1982). Un tel classement reste subjectif avec des risques d'erreur dans l'attribution à l'Italie ou à Lyon, et ne peut être en mesure de distinguer les productions de la Muette d'autres ateliers éventuels. L'attribution à Lyon à partir des analyses de pâtes reste plus fiable mais ne résout pas

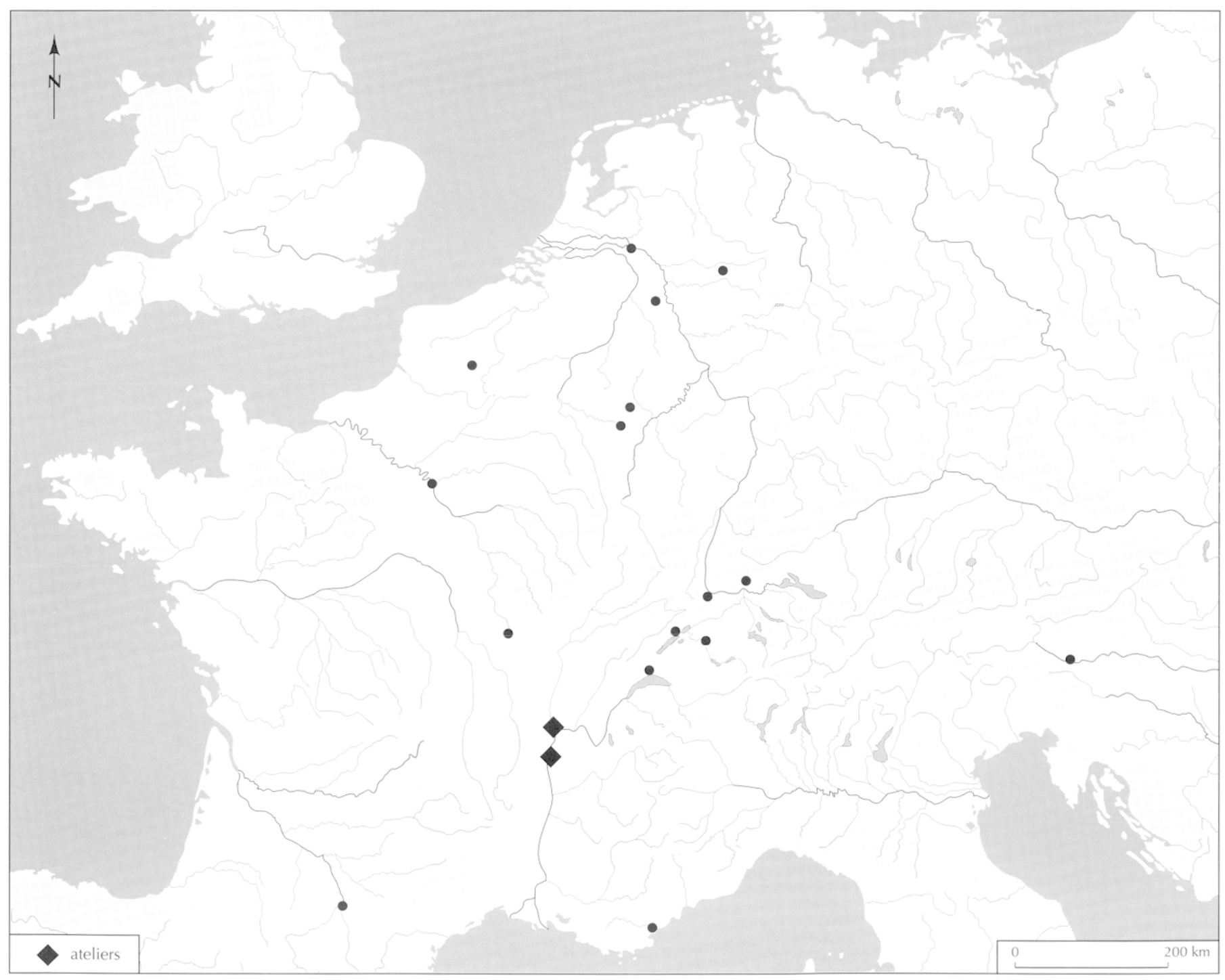

Fig. 94. Carte de répartition des gobelets signés A co et Hilarus Aco. 


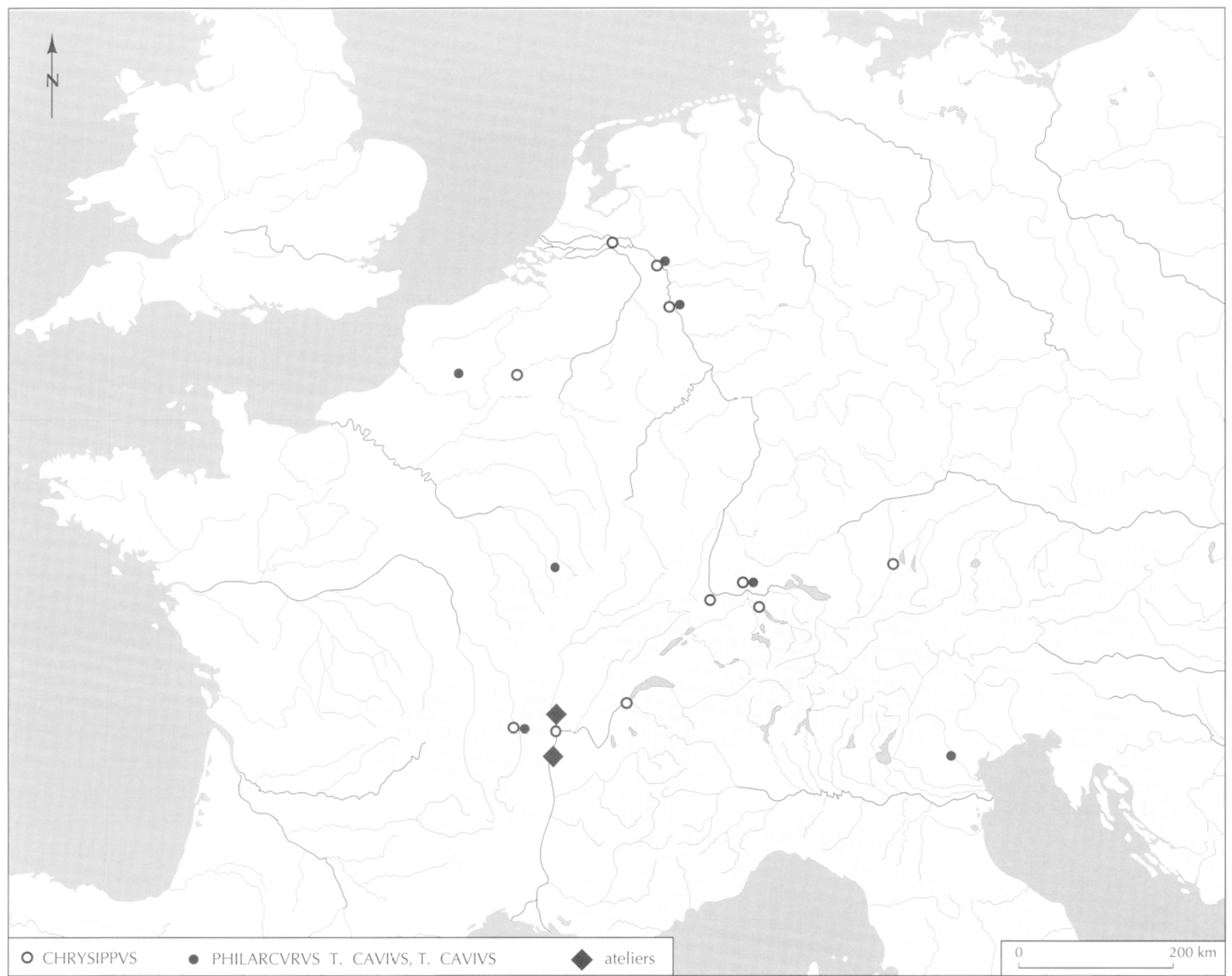

Fig. 95. Carte de répartition des gobelets signés Chrysippos, Philarcorrs et T. Cavius.

totalement la difficulté. Les analyses du matériel de Haltern révèlent une composition lyonnaise pour $50 \%$ de la sigillée, mais la majorité de ce matériel est représenté par les produits d'Ateiss dont rien ne prouve qu'il ait effectivement travaillé à la Muette. À Bavai également (Vanderhoeven, 1989) sur les soixante-douze estampilles recensées, $50 \%$ sont représentés par des ateiana, dont $50 \%$ seraient gaulois.

Depuis la publication de Haltern, la poursuite des recherches sur les compositions des argiles lyonnaises et riennoises a permis en outre d'attribuer à Vienne la marque de Mena Avili dont un seul exemplaire avait été recueilli à la Muette (Lasfargues, 1976, VII, 1). La carte de répartition des estampilles de Mena Avili traduit une diffusion assez large, qui tendrait à prouver que l'atelier de sigillée viennois était plus important qu'on ne le supposait. La découverte dans les fouilles de la Muette de moules et d'un poinçon de composition viennoise démontre en outre que l'atelier de Vienne n'a pas produit que des sigillées lisses.

Concernant la Muette, il faut noter que les productions de la première phase (ensemble I) ne paraissent pas 
avoir connu une grande diffusion; à titre d'exemple, les bols du service II de cette période, pourtant particulièrement faciles à identifier, ne se retrouvent qu'cn quelques rares exemplaires signalés par S. von Schnurbein (1982) et dont la "mauvaise qualité » avait autrefois attiré l'attention de A. Oxé. À Dangstetten, où l'on s'attendrait à trouver les céramiques correspondantes, la part du matériel dont l'origine lyonnaise est possible ou probable ne représente que sept estampilles ( 3 exemplaires C. Senti et 4 L. Thyrsi). Le même phénomène existe à Rödgen (Schönberger, Simon, 1976) et semble-t-il à Oberaden.

Par ailleurs d'autres types de céramiques ne semblent pas connus en dehors de l'atelier, comme les plats à vernis rouge pompéicn ou lcs parois fines à pâte calcaire. Pour l'ensemble II, plus récent, qui correspondrait davantage à l'époque de la grande exportation de l'atelier, on remarque de la même façon un décalage avec le matériel des sites de consommation, en particulier Haltern. Outre la part prépondérante des sigillées d'Ateivs déjà évoquée, on remarque entre l'atelier et Haltern les différences de proportion entre service I et service II. Ce dernier ne représente que $16 \%$ à la Muette, contre $50 \%$ à Haltern.

Il ressort de tout cela que le matériel exhumé dans les fouilles de la Muette ne correspond pas à la grande pćriode de diffusion de l'atelier, qu'il faut situer à une date légèrement plus récente. Il est possible aussi que l'atelier auquel correspond cette large diffusion ne soit pas localisé sur le site même de la Muette, mais sur un site voisin des bords de Saône.

A. D., M. G.

\section{LA COMMERCIALISATION}

L'importation massive de sigillée sur le limes rhénan à partir de 15 avant J.-C. a conduit certains auteurs à supposer que la commercialisation des sigillées ne concernait que les sites militaires et que le réseau commercial avait été organisé dans le but d'approvisionner l'armée. Cette thèse a été combattue à juste raison par $\mathrm{C}$. M. Wells qui, tout en reconnaissant que l'armée avait joué un grand rôle, arguait du fait que : "Once the sigillata started to be imported for military use, it became available on the civilian market. ( (Wells, 1992, p. 195).

Les cartes de répartition des productions lyonnaises montrent que celles-ci ont été commercialisées avant la campagne de Drusus et confirment que leur diffusion a concerné aussi bien les sites civils que militaires. La carte de répartition des gobelets d'Aco de Loyasse (fig. 92) illustre bien ce phénomène, avec leur présence sur des sites comme Bâle, Neuss ou Dangstetten, mais aussi Paris, Liberchies ou le Titelberg et bien que les imitations de sigillées soient plus difficiles à identifier, on peut penser qu'elles ont connu une diffusion comparable.

De même, la carte des productions de la Muette (fig. 93) montre que la sigillée est arrivée de la même façon sur des sites civils, comme le prouvent les pourcentages importants que représente cette céramique sur des sites comme Bavai, Tongres ou même Corseul. Il n'en demeure pas moins vrai que les sites militaires rhénans ont représenté un marché privilégié et qu'en terme de volume ils ont sans doute constitué le principal débouché des ateliers lyonnais. La question est de savoir si les marchés civils ont bénéficié de l'existence d'ateliers créés spécialement pour l'approvisionnement de l'armée ou si, au contraire, l'approvisionnement de l'armée a fait appel à des ateliers créés pour approvisionner la Gaule dans son ensemble.

C. M. W'ells établit une relation entre l'atelier de la Muette et l'atelier monétaire de Lyon, destiné à payer l'armée du Rhin, allant jusqu'à poser la question : "Did the pottery and coins use the same supply routes, share the same riverboats or the same wagons? "(Wells, 1992, p. 200). Il est bien difficile de répondre à cette question. Une réponse affirmative supposerait que l'armée prenait elle-même en charge l'approvisionnement des camps en céramique, ce qui semble difficilement concevable.

L'hypothèse de Wells se fonde sur la quasi-absence de sigillée lyonnaise à Dangstetten, où le monnayage de Lyon est totalement absent, à l'inverse du site d'Haltern où les sigillées et les monnaies de Lyon abondent. Cela n'est pas un argument suffisant pour lier les deux phénomènes. La commercialisation des céramiques lyonnaises ou viennoises a précédé l'essor de l'atelier de la Muette et les émissions à l'autel de Lyon. Il est vrai qu'à partir de 10 avant J.-C., les exportations lyonnaises connaissent un saut qualitatif et quantitatif et que cet essor coincide avec le développement de l'atelier monétaire, mais on peut penser que tout ceci n'est que la conséquence des mutations politiques et économiques, notamment de l'élévation de la colonie de Lyon au rang de Capitale des trois Gaules.

C. M. Wells (1977) a proposé trois modèles de distribution : 
l. Les ateliers de potiers créent leur propre réseau de distribution. Cette hypothèse avait été émise par S. Loeschcke (1909) pour expliquer la grande quantité de produits d'Ateivs à Haltern. Cela suppose que ces firmes arrivent à contrôler le marché.

2. La distribution est le fait d'intermédiaires indépendants, comme les negotiatores artis rei cretariae. Il s'agit de grossistes qui peuvent faire le commerce d'un ou plusieurs produits et peuvent éventuellement aroir leur propre entreprise de transport.

3. L'armée achète et achemine elle-même les produits nécessaires à son approvisionnement. Elle envoie ses intendants faire le tour des ateliers ou des commerçants pour sélectionner les céramiques. C. M. Wells doute qu'un système aussi centralisé puisse exister à l'époque d'Auguste (Wells, 1977, p. 136) et il pense que plus probablement l'approvisionnement de l'armée était organisé par des publicani avec lesquels celleci passait contrat. Cette hypothèse qui, comme le souligne Wells, est compatible avec le rôle d'intermédiaires indépendants, ne paraît pas beaucoup plus plausible que les deux autres modèles. L'idée selon laquelle les officines de potiers créent leur propre réseau de distribution et prennent en charge la commercialisation sur une longue distance n'est pas du tout convaincante. Elle manque sérieusement d'argument.

De même, l'achat et l'acheminement directement par l'armée pourraient se comprendre pour des produits de première nécessité en particulier pour des produits alimentaires, mais on en voit mal la nécessité pour la vaisselle. Cette hypothèse est d'autant moins plausible qu'il n'existe aucune preuve que l'annone ait toujours pris en charge le ravitaillement de l'armée et qu'on ne sait pas comment se faisait l'arrivée des produits autres que le blé : huile, vin, garum (Tchernia, 1986, p. 13).

Dans l'hypothèse de contrats passés entre l'armée et des publicani, ceux-ci ont pu profiter de l'organisation du réseau et des facilités que leur créent les contrats avec l'armée pour organiser leur propre commerce à destination des marchés civils. Toutefois, il est tout aussi possible que l'approvisionnement de l'armée pour des produits qui n'étaient pas de première nécessité n'ait pas fait l'objet de tels contrats mais ait été pris en charge par des negotiatores indépendants. Il est à douter que l'on puisse trouver des éléments permettant de trancher, d'autant que les deux systèmes ont pu coexister.
La mise en évidence d'une production d'amphores à Lyon dès l'époque augustéenne, destinée au conditionnement des vins ou de saumures importées en vrac, constitue un élément nouveau qui modifie la perception que l'on pouvait avoir du commerce lyonnais. Cela implique en effet que Lyon joue un rôle important dans la distribution des produits alimentaires et donc dans le ravitaillement des camps. Il en résulte que la commercialisation des productions céramiques a pu bénéficier d'un réseau commercial organisé d'abord pour ravitailler les sites militaires et que la céramique n'a constitué qu'un produit secondaire dans le volume des échanges. Il est tout à fait possible que des commerçants se soient spécialisés dans le commerce des céramiques, mais il est possible également que des negotiatores importants se soient livré au commerce de différents produits comme le negotiator vinarii et artis cretariae que nous fait connaître une inscription de Lyon (CIL, XIII, 2033).

A. D.

\section{LA CHRONOLOGIE DES ATELIERS LYONNAIS}

Les études préliminaires consacrées aux ateliers lyonnais avaient conduit à dater l'activité de l'atelier de Loyasse entre 30 et 15 avant J.-C. et celle de l'atelier de la Muette entre 15 avant et 15 après J.-C. (Lasfargues, 1973). Ces datations avaient été proposées à partir de la chronologie relative évidente entre ces deux ateliers, et de la comparaison avec les sites militaires du limes, Haltern en particulier. L'étude détaillée du mobilier issu de ces fouilles oblige toutefois à corriger partiellement ces estimations. La date avancée pour l'activité de l'atelier de Loyasse demeure inchangée : 30-15 avant J.-C. En revanche, il ressort de l'étude typologique et statistique des productions sigillées de la Muette un décalage certain entre le faciès de ce matériel et celui du camp de Haltern. L'étude conjointe des différentes catégories de céramique produites par l'atelier permet donc aujourd'hui de proposer d'autres dates pour les ensembles céramiques de la Muette qui doivent se situer probablement entre 2015 et $10-5$ avant J.-C.

Plus délicate est la datation des deux ensembles mis en évidence par l'étude. Le manque de références sur les sites de consommation, proches ou lointains, rend difficile l'estimation de la durée de la première période 
(ensemble I) qui peut se situer entre 20 et 15 avant J.-C. Quant à l'ensemble II, son décalage avec le faciès de Haltern ainsi que sa faible représentation à Neuss et Dangstetten tendraient à le situer entre 12 et 5 avant J.-C.

Il ne faudrait pas déduire pour autant de ces résultats que l'activité des ateliers lyonnais précoces s'est limitée à la période comprise entre 30 et 10 avant J-C. Concernant le début des productions lyonnaises, les découvertes du quai Saint-Vincent laissent supposer l'existence d'ateliers antérieurs à celui de Loyasse. La datation des fours de Saint-Vincent reste toutefois sujette à discussion et leur production est mal assurée. Il ressort malgré tout des analyses réalisées sur les céramiques à vernis noir ou rouge du type de celles de Loyasse qu'il existe d'autres groupes de production dont les compositions sont proches de celles de la Muette. Tout ceci concourt à supposer l'existence d'une phase de production plus ancienne des ateliers des rives de la Saône, qui aurait précédé l'atelier de la Muette et pourrait être contemporaine, voire plus ancienne que l'atelier de Loyasse.

Pour la production de sigillée, le constat est identique : la période de fabrication des sigillées lyonnaises est plus large que ne le montre l'image fournie par le site de la Muette, le matériel de l'atelier ne correspondant pas à la grande diffusion de sa production représentée notamment sur le site de Haltern. Qu'elle provienne de l'atelier de la Muette ou d'un site d'atelier voisin, la production de sigillée non seulement se poursuit, mais encore se développe après 10 avant J.-C. La question est de savoir si elle se prolonge au-delà de 10 après J.-C. et à quelle date elle cesse. Un certain nombre de sites plus récents qu'Haltern fournissent des ensembles dans lesquels figurent encore des productions lyonnaises associées aux sigillées de la Gaule du Sud.

Si l'on se réfère aux fouilles régionales, les productions lyonnaises se rencontrent encore dans les niveaux datés des années 15-20 après J.-C. À Saint-Romain-en-Gal, une étude réalisée sur le mobilier des ensembles augustéens de la maison des Dieux Océan a montré que les productions régionales n'apparaissent pas avant l'horizon SRG 3 qui voit apparaître également les premières importations de la Graufesenque (Desbat, Picon, 1992). Les contextes lyonnais, notamment sur les sites du VerbeIncarné et de la rue des Farges, permettent les mêmes constatations. Même si l'on admet qu'une partie de ce matériel est résiduel, il apparaît que la diffusion locale des produits lyonnais se situe surtout au début de notre ère, postérieurement à l'horizon de Haltern.
Tout se passe comme si la production de sigillée des officines lyonnaises avait été tournée uniquement vers l'exportation et que la diffusion locale ne se soit développée qu'avec le déclin des ateliers.

A. D.

\section{L'ARRÊT DES ATELIERS DE SIGILLÉE}

L'arrêt des productions de la Muette est une des questions non résolues. Les données que nous possédons permettent d'établir que la production s'interrompt au tout début du règne de Tibère. Les dates proposées par $\mathrm{S}$. von Schnurbein (1982, p. 20), entre 10 avant et 10 après J.-C. avec un floruit à partir de 5 avant J.-C., peuvent être élargies. Dans la région lyonnaise, les produits de la Muette apparaissent tardivement. On les rencontre surtout dans les contextes du début $\mathrm{du} \mathrm{I}^{\mathrm{cr}} \mathrm{s}$. associés aux premières importations de la Gaule du Sud (Desbat, Picon, 1992). Faut-il voir là la traduction d'un repli sur une clientèle régionale? Un tel phénomène a été souvent constaté; la phase de déclin d'un atelier se traduisant par une diminution, voire un arrêt des exportations et un retour à une diffusion locale avant un arrêt complet. Ce constat ne résout pas le problème posé par l'arrêt des ateliers de sigillée lyonnais et la principale question reste de savoir quelles sont les raisons de leur déclin.

La question se pose dans des termes voisins avec celle du déclin des ateliers italiques, ou celle du déclin de la Graufesenque et de l'essor de Lezoux : le déclin de la Muette est-il la conséquence du développement et de la concurrence des ateliers de la Graufesenque ou bien au contraire l'essor de la Graufesenque a-t-il été rendu possible par le déclin des ateliers lyonnais? Il faut bien constater que la réponse à cette question n'est pas des plus faciles.

L'installation à Lyon d'ateliers destinés à alimenter le nord de la Gaule et le limes paraissait correspondre à une logique commerciale rigoureuse. La situation de ces ateliers sur l'axe rhodanien semblait privilégiée compte tenu des possibilités offertes par le réseau routier et plus encore par le réseau fluvial. La localisation à Iyon aurait permis d'approvisionner encore plus facilement le sud de la Gaule. Malgré cela, on constate un déclin brutal des officines de sigillée lyonnaise et l'essor, peu de temps après ou au même moment, des ateliers de la Graufesenque dont la situation géographique peut sem- 
bler moins favorable. Les quelques données que nous possédons sur les ateliers de sigillée montrent que la cuisson à température élevée dans des fours à tubulures nécessitait un combustible associant une forte chaleur avec des flammes longues et que les résineux (pin ou sapin) étaient ainsi un des combustibles utilisés de préférence pour la cuisson de sigillées (Vernhet, 1981 et 1993).

Faut-il concevoir qu'une des raisons, sinon la principale, du déclin des ateliers lyonnais a été un tarissement des ressources en combustible? Il est en effet possible qu'après deux ou trois générations les ressources en résineux n'aient plus été suffisantes pour permettre le maintien et, plus encore, le développement de grandes officines de sigillée, dont la consommation était considérable $^{17}$. Les calculs établis pour la Graufesenque estiment la consommation moyenne des grands fours à 40 tonnes de bois et celle de l'atelier, pour une quarantaine d'années seulement, à 90000 tonnes de bois soit l'équivalent de 10000 hectares (Vernhet, 1993). On a évoqué la possibilité d'acheminer du bois par flottage, mais outre qu'un tel système est réservé à des bois longs, qui n'étaient pas le combustible habituel des potiers, il ê̂t sans doute représenté un surcoût important.

Ce problème de combustible pourrait expliquer le constat de la disparition ou de la limitation en taille des ateliers urbains au profit de l'installation de gigantesques centres de production en milieu rural, dont la Graufesenque et Lezoux sont des exemples au même titre que Jaulges, Villiers- Vineux ou l'Argonne.

17. Il faut préciser que cette disparition des ressources en combustible ne serait pas imputable aux seuls poticrs mais que la consommation de bois pour la construction y est surement darantage pour quelque chose.
Le déclin et la disparition des ateliers lyomnais de sigillée, bien que relativement rapides, ont dî quand même s'échelonner sur quelques années. Il est possible que la production se soit maintenue jusque vers les années 15-20 après J.-C., mais avec une production de qualité médiocre et une diffusion purement locale. Il faut rappeler que ce déclin ne frappe que les sigillées puisque la production de céramiques à parois fines va se maintenir de même que d'autres productions, en particulier celle des amphores.

Parmi les questions qui subsistent, il faut évoquer le devenir des potiers qui travaillaient dans ces officines. L'étude des ateliers modernes traditionnels permet d'observer des constantes dans le phénomène de déclin des ateliers :

- reconversion d'une partie des potiers vers d'autres activités,

- déplacement d'une autre partie vers d'autres centres (généralement les potiers les plus qualifiés),

- maintien d'une activité réduite, avec baisse de plus en plus marquée de la qualité et diffusion de plus en plus restreinte.

Ce schéma pourrait fort bien s'appliquer aux ateliers lyonnais de sigillée. Il est probable qu'une partie des potiers a du se déplacer vers d'autres centres. On pense en premier lieu à Lezoux, où le début d'une production de type sigillée intervient à cette époque et dont les rapports avec Lyon ont été souvent évoqués. Il est également possible, voire très probable, qu'une autre partie des potiers se soit déplacée à la Graufesenque. La présence d'une officine d'Ateivs pourrait donner à penser que celle-ci a joué un rôle dans l'introduction de la technique de la sigillée à la Graufesenque.

A. D. 